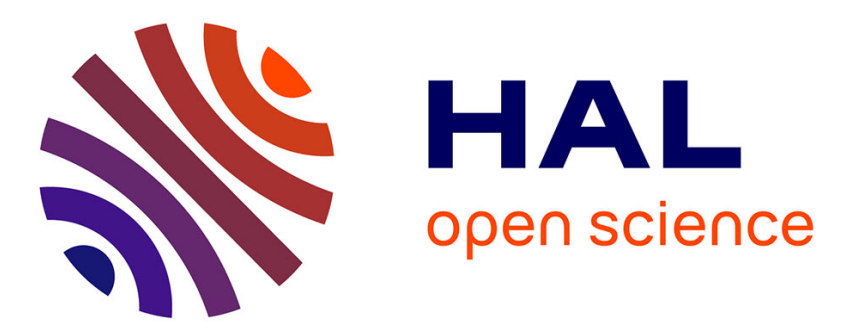

\title{
Beyond the limitations of perturbation methods for real random eigenvalue problems using Exceptional Points and analytic continuation
}

\author{
Martin Ghienne, Benoit Nennig
}

\section{To cite this version:}

Martin Ghienne, Benoit Nennig. Beyond the limitations of perturbation methods for real random eigenvalue problems using Exceptional Points and analytic continuation. Journal of Sound and Vibration, 2020, 480, pp.115398. 10.1016/j.jsv.2020.115398 . hal-02536849

\author{
HAL Id: hal-02536849 \\ https://hal.science/hal-02536849
}

Submitted on 8 Apr 2020

HAL is a multi-disciplinary open access archive for the deposit and dissemination of scientific research documents, whether they are published or not. The documents may come from teaching and research institutions in France or abroad, or from public or private research centers.
L'archive ouverte pluridisciplinaire HAL, est destinée au dépôt et à la diffusion de documents scientifiques de niveau recherche, publiés ou non, émanant des établissements d'enseignement et de recherche français ou étrangers, des laboratoires publics ou privés. 


\title{
Beyond the limitations of perturbation methods for real random eigenvalue problems using Exceptional Points and analytic continuation
}

\author{
Martin Ghienne ${ }^{\mathrm{a}}$, Benoit Nennig, ${ }^{\mathrm{a}, *}$ \\ ${ }^{a}$ Institut supérieur de mécanique de Paris (SUPMECA), Laboratoire Quartz EA 7393, \\ 3 rue Fernand Hainaut, 93407 Saint-Ouen, France.
}

\begin{abstract}
A numerical method is proposed to approximate the solution of parametric eigenvalue problem when the variability of the parameters exceed the radius of convergence of low order perturbation methods. The radius of convergence of eigenvalue perturbation methods, based on Taylor series, is known to decrease when eigenvalues are getting closer to each other. This phenomenon, knwon as veering in structural dynamics, is a direct consequence of the existence of branch point singularity in the complex plane of the varying parameters where some eigenvalues are defective. When this degeneracy, referred to as Exceptional Point $(\mathrm{EP})$, is close to the real axis, the veering becomes stronger.

The main idea of the proposed approach is to combined a pair of eigenvalues to remove this singularity. To do so, two analytic auxiliary functions are introduced and are computed through high order derivatives of the eigenvalue pair with respect to the parameter. This yields a new robust eigenvalue reconstruction scheme which is compared to Taylor and Puiseux series. In all cases, theoretical bounds are established and all approximations are compared numerically on a three degrees of freedom toy model. This system illustrate the ability of the method to handle the vibrations of a structure with a randomly varying parameter. Computationally efficient, the proposed algorithm could also be relevant for actual numerical models of large size, arising from other applications involving parametric eigenvalue problems, e.g., waveguides, rotating machinery or instability problems such as squeal or flutter.
\end{abstract}

Keywords: Uncertainty propagation, Veering, Exceptional point, Puiseux series, parametric eigenvalue problem, defective eigenvalue

\section{Introduction}

The prediction of the vibrational behaviour of structure or machinery allows to design and optimize solutions for noise and vibration reduction from early design stage. Moreover, considering the variability of the actual behavior of a structure allows to design more robust solutions. For usual industrial structures, finite element models with large number of degree-of-freedom are usuelly required, besides, when the system have varying or uncertain parameters, the characterization of its vibrational behaviour becomes even more challenging. This implies high computational costs to solve the associated eigenvalue problem.

Uncertainty propagation is mainly addressed using two frameworks. On the one hand, non-probabilistic approaches allow to handle random and epistemic uncertainties. They include for instance interval approaches, fuzzy logic [1] or lack-of-knowledge theory [2-4]. On the other hand, probabilistic approaches aim to model the natural variability of random phenomena. Such approaches can be further divided into parametric and non-parametric approaches [5]. While the later allows to consider measurement or modeling uncertainties, parametric methods only consider the variability of the model parameters. This latter framework is retained for this work. In the context of computational mechanics, the most common parametric

\footnotetext{
*Corresponding author

Email addresses: Martin.Ghienne@supmeca.fr (Martin Ghienne), benoit.nennig@supmeca.fr (Benoit Nennig)
} 
probabilistic method is the Stochastic Finite Element Method (SFEM). The SFEM is based on the classical deterministic Finite Element Method and consider the elements properties as random [6]. It is mainly divided into three main approaches: statistical approaches, also referred to as Monte-Carlo Simulations (MCS) [7, 8], perturbation methods [9]11 and the Spectral Stochastic Finite Element Method (SSFEM) [6. 12, 15].

MCS are the most straightforward methods. They consist in sampling the input random parameters and solving each associated configurations of the deterministic model to generate samples of the output random quantities. Even if they converge to the exact solution of the random problem, their major drawback is their rate of convergence. Numerous techniques, generally based on variance reduction or on computation parallelization, have been developed [11, nevertheless these methods remain inadequate for structural analysis. Initiated by Wiener's works [16], the SSFEM was introduced by Ghanem and Spanos [12]. It is based on the discretization of the random quantities among a finite random space. The random output of the system is then represented using orthogonal polynomials of the input random variables called the Polynomial Chaos (PC). Even if efforts have been made to reduce the computational cost of PC methods [14, 17, SSFEM still requires intensive computations when high-order polynomials or a high number of random parameters are involved. Polynomial Chaos expansion can be identified as a surrogate model (also referred to as metamodel [18]) to be substituted to the original model to decrease computational costs. Other surrogate models like quadratic response surfaces or Kriging can be found in the litterature [19]. Common to all these models is to be built from a limited number of runs of the original model. As all interpolation techniques, the convergence is sensitive to the smoothness of the original model and may be less efficient in the vicinity of spectral degeneracies [20. Finally, perturbation approaches provide a framework both straightforward and easy to implement to reduce the computational cost of MCS. It consists in the approximation of the random quantities using Taylor (or Neumann) expansion [9 11, 21]. They are usually limited to first or second order and have been applied to a large field of applications [22]. The first order Taylor expansion allows to obtain a closed-form expression of the joint probability density function of the random eigenvalues when input random variables are assumed to be Gaussian [10]. If random eigenvalues cannot be modeled as Gaussian random variables, perturbation methods (if necessary using higher order expansion) allows to approximate statistical moments of the random eigenvalue in order to identify their probability density function (pdf) [9]. These approaches implicitly requires small variability of the random parameters in order to be able to describe it as perturbations around their nominal value. It is commonly assumed that perturbation approaches are restricted to input random variables with coefficient of variation smaller than 0.15 [23, 24] or less when eigenvalues are getting close to each other 9]. This constitutes the major limitation of perturbation methods and is emphasized by the lack of intrinsic quantitative criterion to a priori define a "small variability" of a parameter and thus the validity domain of these methods. To tackle random parameter with larger variability, it is possible to carefully choose the expansion point [9] or to consider a first order Taylor expansion of the structure eigenvectors to estimate random eigenfrequencies [25. This yields a non-intrusive and scalable approach to approximate the random eigenvalues with a better accuracy than the classical second order perturbation technique while reducing the computational costs.

This study focuses on perturbation methods for real random eigenvalue problems. It aims to identify the validity domain of such approaches and to extend it through analytic continuation. Eigenvalue perturbation is a widely spread mathematical problem [20, 26] arising in many fields of application, ranging from noise attenuation in waveguides [27] to fluid-structure interaction like flutter [28, 29]. These problems are inherently parametric and the behavior of eigenvalue loci, when parameters varies, has been widely studied for these applications in a deterministic framework.

The parametric study of the eigenvalues of an hermitian system shows that, some eigenvalues may approach to each other, without crossing, and then veer away, depending on the parameter value. Concurrently, the involved eigenvectors swap. This phenomenon is referred to as the veering phenomenon [30-32] in structural dynamics. In a stochastic context, the radius of convergence of perturbation methods is known to decrease when eigenvalues are getting closer to each other [9]. Although the literature has few criteria to qualify the veering intensity [33, 34, the validity domain of perturbation methods for random eigenvalues is hardly predictable. The veering is known to be a consequence of the existence of branch point singularity 31, where eigenvalue coalesce, in the complex plane of the varying parameters referred to as Exceptional Point (EP) [20]. When the parameter is extended from real axis to the complex plane, the eigenvalue problem 
becomes non-Hermitian and this kind of system has recently attracted a large attention in classical and in Quantum Physics when losses, gain or Parity-Time symmetry [35] are introduced.

The key idea of this paper is to use non-Hermitian framework and analytic continuation around the EP, introduced in [36], to go beyond the standard limitations of perturbation methods. For the sake of simplicity, a single varying parameter is considered, but the results could be extended to multi-parametric systems.

The paper is organized as follows. Section 2 recalls the random generalized eigenvalue problem and presents a three degrees of freedom (dof) toy model system [9, 37] used as a guideline example throughout the paper. Then, section 3 provides a scalable and computationally efficient way to get high order eigenvalues derivatives and the associated Taylor series with its radius of convergence. The analytic continuation of the eigenvalue loci in the vicinity of the EP using the Puiseux series and analytic auxiliary functions is then investigated in section 4 . Finally, the section 5 compares the proposed approximation and provides an algorithm to reconstruct the eigenvalue loci over an extended range of the system input parameter in comparison with standard perturbation approaches.

\section{Stochastic eigenvalue problem}

\subsection{Problem statement}

The random eigenfrequencies characterization of an undamped (or proportionally damped) structure with random parameters requires to solve a discrete random eigenvalue problem arising, for instance, from the finite element method discretization of the continuous system. The random generalized parametric eigenvalue problem, fulfills by the $\mathcal{N}$ degrees of freedom (DoF) of the discretized structure, is defined by

$$
\left[\mathbf{K}(\nu(\theta))-\lambda_{i}(\nu(\theta)) \mathbf{M}(\nu(\theta))\right] \mathbf{\Phi}_{i}(\nu(\theta))=\mathbf{0}
$$

where $\nu(\theta)$ is the random parameter of the structure standing for material properties and $\theta \in \Omega$ is a basic event from the complete probability space $\Omega$. The real symmetric matrices $\mathbf{M}(\nu(\theta)) \in \mathbb{R}^{\mathcal{N} \times \mathcal{N}}$ and $\mathbf{K}(\nu(\theta)) \in \mathbb{R}^{\mathcal{N} \times \mathcal{N}}$ are the mass and stiffness matrices of the structure and their randomness is inherited from the random parameters $\nu(\theta)$. The random eigenvalues, corresponding to the square of the resonance frequency, and the associated random eigenvectors of the dynamic system are denoted by $\lambda_{i}(\nu(\theta))(i=$ $1, \ldots, \mathcal{N})$ and $\boldsymbol{\Phi}_{i}(\nu(\theta)) \neq \mathbf{0}$, respectively. To alleviate notations, the randomness of the different quantities will be implicitly handled by $\nu$ in the following. Random eigenvalues are modeled as random variables characterized by their joint pdf. Computing the statistical moments of the eigenvalues allows to synthesize statistical realizations to estimate their pdf.

Without loss of generality, we assume that the mass properties of the structure are better handled than stiffness properties, thus the mass matrix $\mathbf{M}$ is assumed to be deterministic.

\subsection{A three Dof toy model system}

To illustrate the performances and the validity range of perturbation methods, computation will be performed on a simple undamped three-DoF system. This example, taken from the literature [9, 37], is presented in Fig. 1. Its mass and stiffness matrices are

$$
\mathbf{M}=\left[\begin{array}{ccc}
m_{1} & 0 & 0 \\
0 & m_{2} & 0 \\
0 & 0 & m_{3}
\end{array}\right] \quad \text { and } \quad \mathbf{K}=\left[\begin{array}{ccc}
k_{1}+k_{4}+\nu & -k_{4} & -\nu \\
-k_{4} & k_{2}+k_{4}+k_{5} & -k_{5} \\
-\nu & -k_{5} & k_{3}+k_{5}+\nu
\end{array}\right]
$$

Here, the random parameter $\nu$ corresponds to the stiffness of the central spring, between $m_{1}$ and $m_{3}$. Its support is positive, its expectation and its coefficient of variation are given by $\mu=\mathrm{E}[\nu]=1 \mathrm{~N} \mathrm{~m}^{-1}$ and $\delta[\nu]=\frac{\sigma_{\nu}}{\mathrm{E}[\nu]}=0.2$ (where $\sigma_{\nu}$ stands for the standard deviation of $\nu$ ). Thus, the random parameter $\nu$ can be modeled as random variables with Gamma distribution $\Gamma(\alpha, \beta)[5]$ where the coefficients $\alpha$ and $\beta$, are given by $\mathrm{E}[\nu]=\alpha \beta$ and $\operatorname{Var}[\nu]=\alpha \beta^{2}$.

It is assumed that other stiffnesses are deterministic and respectively equal to $k_{1}=1 \mathrm{Nm}^{-1}, k_{2}=$ $2 \mathrm{Nm}^{-1}, k_{3}=3 \mathrm{Nm}^{-1}, k_{4}=1 \mathrm{Nm}^{-1}, k_{5}=1 \mathrm{Nm}^{-1}$. The masses are also deterministic and equal to 


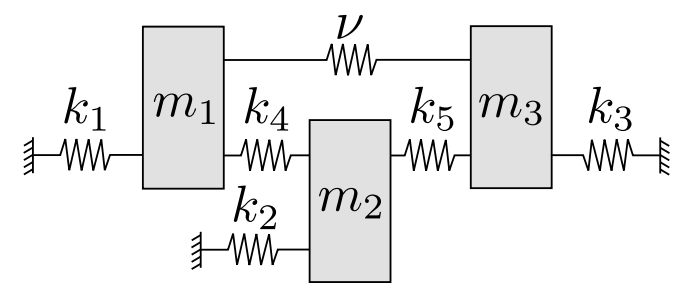

Figure 1: Sketch of the three-DoF undamped spring-mass system.

$m_{i}=1 \mathrm{~kg}$ with $i=1,2,3$. It is known from Ref. 38 that systems with a high degree of symmetry is more prone to veering. Here, the masses configuration is symmetric, and the tuning parameter $k_{3}$ will be changed from its nominal to reinforce or attenuate the veering effect, when specified.

A Monte-Carlo reference simulation (MCS) has been performed to characterize the marginal density function of the three random eigenvalues of the 3-Dof system. Thus 30000 independent samples of the stiffness $\nu$ have been used to obtained the marginal pdf of all the eigenvalues. The results are presented in Fig. 2 in combination with the eigenvalue loci with respect to $\nu$ for low veering to strong veering configurations. Here, the eigenvalues are labeled by ordering their magnitude in ascending order at the nominal value of the parameter. When the veering intensified, it can be observed that the asymmetry of the random eigenvalues marginal pdf strongly increases. In this case, the transformation which yields the pdf of the random eigenvalues from the input random parameter distribution becomes highly non-linear. This illustrates the limitation of first order perturbation methods to small variations when veering occurs between eigenvalues.

A precise knowledge of the mapping $\lambda_{i}(\nu)$, ie the eigenvalue loci, is of primary importance because it can be used to quickly generate the realizations of the random eigenvalues from the realizations of the input parameter. Building such mapping could be related to meta-models approaches [18, 19]. In the next section, the potentiality of higher order perturbation methods to accurately map eigenvalue loci will be investigated.

\section{High order Taylor expansion of the eigenvalues}

The general way to get accurate approximation of the eigenvalue loci is to sample the parametric space and use interpolation [18, altough several eigenvalue problems have to be solved. To the authors best knowledges, no attempt was made to use local approximation based on higher order derivatives. Following results of [39, Theorem 2.1], it is assumed that the operator from Eq. (1) is an analytic function of both $\nu$ and $\lambda$ in the neighborhood of $\nu_{0}$. Then an eigenvalue $\lambda_{i}$ and its associated eigenvector are also analytic in a neighbourhood of $\nu_{0}$, and can be expressed as a $N$-order truncated Taylor expansion around $\nu_{0}$

$$
T_{\lambda_{i}}(\nu)=\sum_{n=0}^{N} t_{n}\left(\nu-\nu_{0}\right)^{n}, \quad \text { where } t_{n}=\left.\frac{\lambda_{i}^{(n)}(\nu)}{n !}\right|_{\nu=\nu_{0}} .
$$

This expansion holds as long as the eigenvalue of the problem (1) are simple. To practically compute such truncated Taylor series, the high order derivatives of the few monitored eigenvalue are required and efficient computational framework exist to obtained them recursivelly.

\subsection{Higher order eigenvalues derivatives computation}

Structural dynamic problems arising in sec. 2 are generally real symmetric and positive definite. Here, these assumptions are relaxed for consistency with next section (see Appendix Appendix A for the Hermitian version). Let us consider a general non-Hermitian eigenvalue problem depending on one complex parameter $\nu$. This eigenvalue problem is represented by a $\mathcal{N} \times \mathcal{N}$ matrix valued function $\mathbf{L}\left(\lambda_{i}(\nu), \nu\right) \equiv \mathbf{K}(\nu)-\lambda_{i}(\nu) \mathbf{M}$, such as

$$
\mathbf{L}\left(\lambda_{i}(\nu), \nu\right) \boldsymbol{\Phi}_{i}(\nu)=\mathbf{0}
$$

Here $\lambda_{i}(\nu)$ and $\boldsymbol{\Phi}_{i}(\nu) \neq \mathbf{0}$ are the $i^{\text {th }}$ eigenvalue and associated right eigenvector. Computing eigenvalue and eigenvector derivatives have received a particular attention over the last decades. Several techniques 

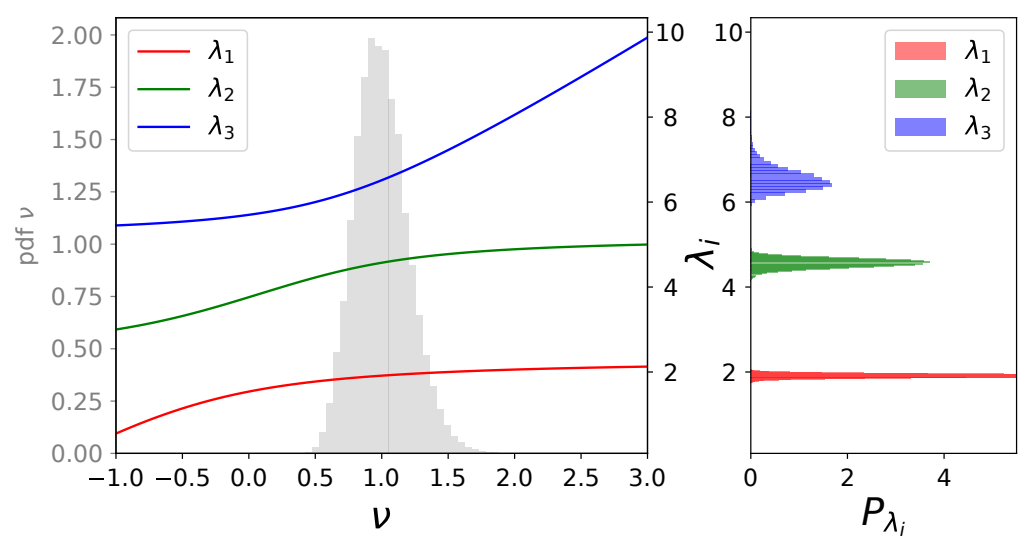

(a) Weak veering, $k_{3}=4, \nu^{*} \approx 0.6341+1.032 \mathrm{i}$.
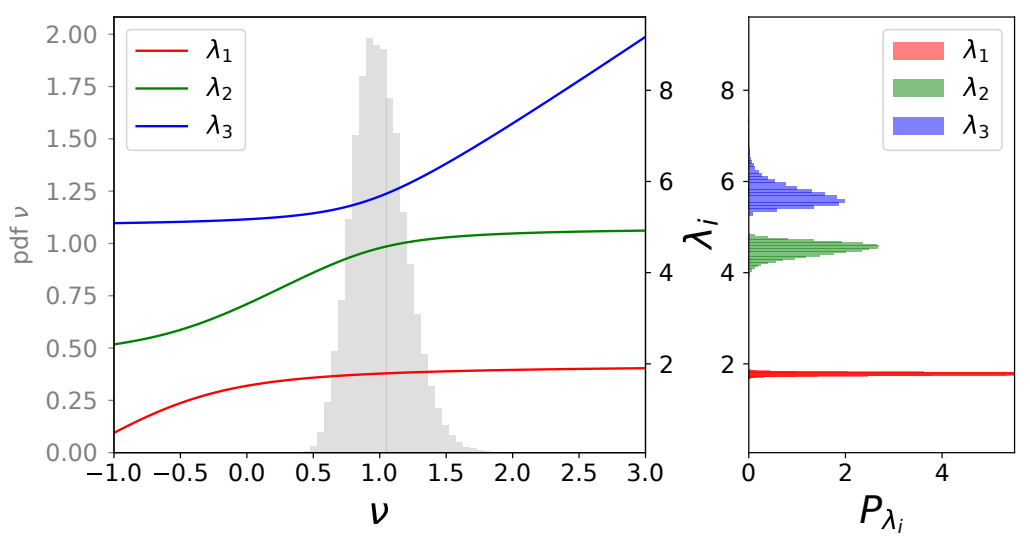

(b) Mild veering, $k_{3}=3, \nu^{*} \approx 0.8926+0.5977 \mathrm{i}$.

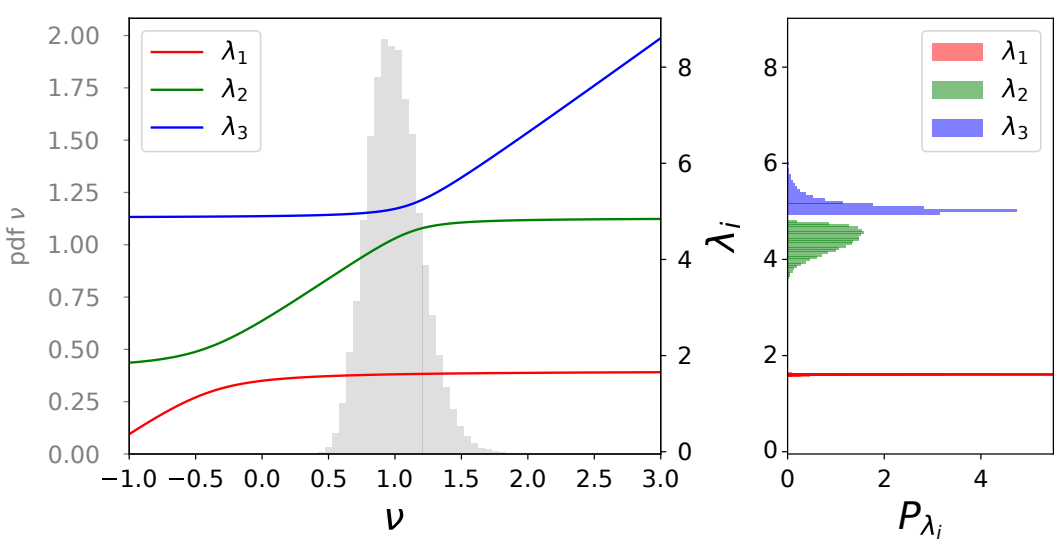

(c) Strong veering, $k_{3}=2.1, \nu^{*} \approx 1.147+0.2600 \mathrm{i}$.

Figure 2: Impact of the veering phenomenon on the marginal density function of the random eigenvalues of the 3-Dof system. 
have been developed depending on the required order of derivative and on the number of studied eigenvalue. See for instance Murthy's survey [40] dedicated to complex non-Hermitian matrices. In the context of this work, the direct method proposed by Andrew et al. 39] based on the bordered matrix, is adopted as in [36]. It is noteworthy that this method can be used to obtained the eigenvalue derivatives with respect to several variables 39 .

As the eigenvector are defined up to multiplicative constant, the derivative of the eigenvector cannot be obtained uniquely as long as a normalization condition has not been applied. For convenience, we define the following norm

$$
\mathbf{v}^{t} \boldsymbol{\Phi}_{i}(\nu)=1
$$

where $\mathbf{v}$ is a constant vector (see [39] and Appendix Appendix A for other normalization like the M-norm) and the superscript ${ }^{t}$ denotes the transposition.

The successive derivatives of the selected eigenvalue $\lambda_{i}$ can be recursively obtained by solving the linear system built on the $n^{\text {th }}$ derivative of the eigenproblem (1) and the normalization condition (5) at $\nu=\nu_{0}$

$$
\left[\begin{array}{cc}
\mathbf{L} & \partial_{\lambda} \mathbf{L} \boldsymbol{\Phi}_{i} \\
\mathbf{v}^{t} & 0
\end{array}\right]\left(\begin{array}{l}
\boldsymbol{\Phi}_{i}^{(n)} \\
\lambda_{i}^{(n)}
\end{array}\right) \equiv\left(\begin{array}{c}
\mathbf{F}_{n} \\
0
\end{array}\right)
$$

where the right hand side (RHS) $\mathbf{F}_{n}$ contains all the other terms arising from the successive derivatives of the considered eigenpairs. The last row of the RHS is zero due to the chosen norm (5) since $\mathbf{v}$ is a constant vector, this normalization choice allows to simplify the recurrence relation. For generalized eigenvalue problem, commonly encountered in structural dynamics, closed form expression of the RHS can be obtained with the Liebnitz' rule for product derivation

$$
\left(\mathbf{K}-\lambda_{i} \mathbf{M}\right) \boldsymbol{\Phi}_{i}^{(n)}-\mathbf{M} \boldsymbol{\Phi}_{i}^{(0)} \lambda_{i}^{(n)}=\mathbf{F}_{n} \equiv-\sum_{k=1}^{n}\left(\begin{array}{l}
n \\
k
\end{array}\right) \mathbf{K}^{(k)} \boldsymbol{\Phi}_{i}^{(n-k)}+\mathbf{M} \sum_{k=1}^{n-1}\left(\begin{array}{l}
n \\
k
\end{array}\right) \lambda_{i}^{(k)} \boldsymbol{\Phi}_{i}^{(n-k)} .
$$

with the convention $\boldsymbol{\Phi}_{i}^{(0)}=\boldsymbol{\Phi}_{i}$ and $\lambda_{i}^{(0)}=\lambda_{i}$. This approach 39 requires to factorize the operator $\mathbf{L}$ for each eigenmode of interest. Nevertheless, it is the most computationally intensive step and the computation of the RHS is generally faster. Its computational complexity depend on the number of non-zeros in the operator derivative and the length of the recurrence (see sec. 5.4 for further details).

\subsection{Radius of convergence and Exceptional Point location}

Let us first study the validity range of the Taylor expansion with the approximation order. We consider the 3-Dof system with all its stiffnesses taken at their deterministic values (see sec. 2.2) and we assume that $\nu$ is varying about $\nu_{0}=\mu=1 \mathrm{~N} \mathrm{~m}^{-1}$. The Taylor expansions of the three eigenvalues of the problem, obtained with (6), are presented Fig. 3 for different approximation orders $N \in \llbracket 1,20 \rrbracket$. When no veering occurs, see for instance the first eigenvalue $\lambda_{1}$ or the other when $\nu_{0} \gg 1 \mathrm{~N} \mathrm{~m}^{-1}$, the series converge quickly and only few terms are required to reconstruct the eigenvalue on a wide stiffness range. If a moderate veering is present, like between $\lambda_{2}$ and $\lambda_{3}$, the Taylor expansion perfectly fits these eigenvalues about the point $\nu_{0}$ but diverges over a certain value of $\nu$ regardless of the approximation order. This illustrates the strong influence of the veering on the convergence of the Taylor series. To quantify this impact, the veering between $\lambda_{2}$ and $\lambda_{3}$ can be tuned by varying the stiffness parameter $k_{3}$ like in Fig. 2. The Fig. 4 illustrates the Taylor series reconstruction of the eigenvalues for weak, mild and strong veering conditions. It can be noticed that the radius of convergence of the Taylor expansion decreases when the veering increases. This behavior reveals the presence of a singularity in the parameter complex-plane [41, sec. 5.4].

For hermitian problems, it can be shown that the veering phenomenon is a consequence of the eigenvalue problem degeneracy for a complex value of the parameter [31, 38, 42. Indeed, if the parameter of the problem is extended in the complex-plane, the $\mathbf{K}$ (and $\mathbf{M}$ ) matrices becomes non-Hermitian and eigenvalues and eigenvectors could coalesce. The coalescing point in the complex space is referred to as Exceptional Point (EP) [20] and is denoted by $\nu^{*}$. At the EP, two or more eigenvalues are defective and the matrix cannot be diagonalized.

To illustrate that, the evolution of the real parts of $\lambda_{2}$ and $\lambda_{3}$ are given in Fig. 5 when the parameter $\nu$ is extended in the complex-plane. It can be observed that the different eigenvalues are in fact different 


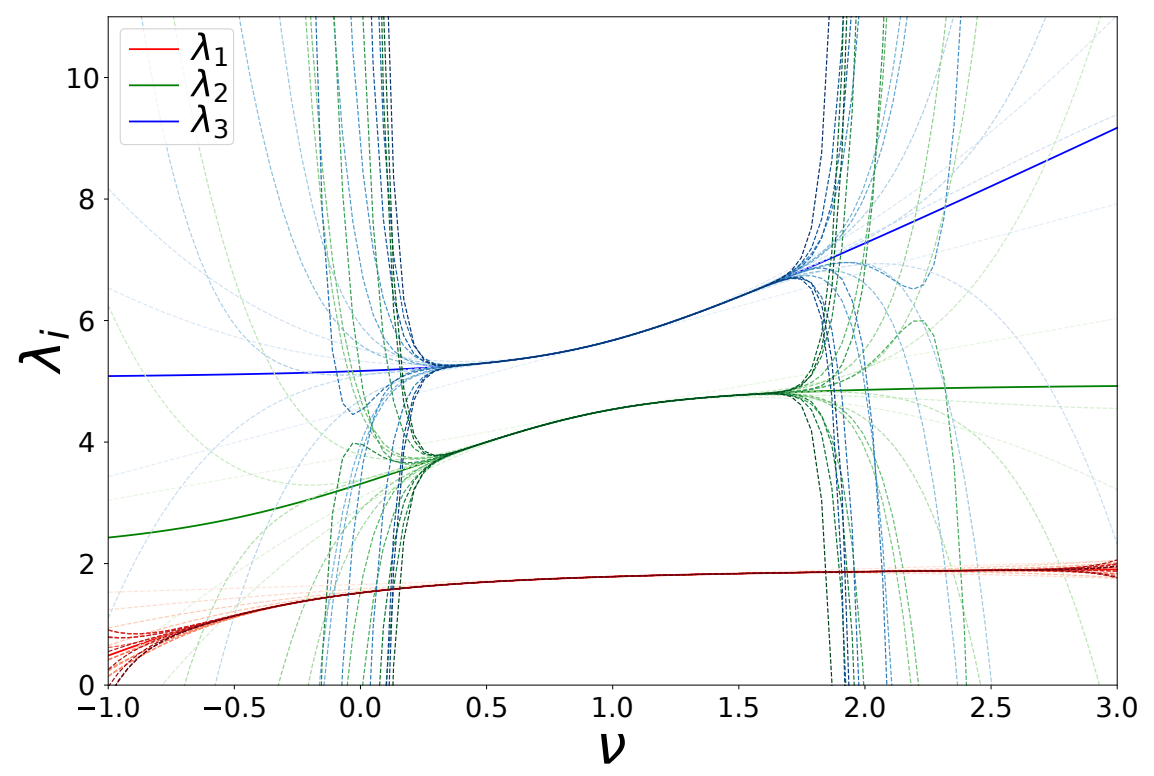

Figure 3: Impact of the order of the Taylor expansion $(n \in \llbracket 1,20 \rrbracket)$ on the eigenvalue approximation (dashed lines) around $\nu_{0}=1 \mathrm{~N} \mathrm{~m}^{-1}$ for $k_{3}=3$. The darkest colors correspond to highest degree of approximation.

branches of the same Riemann surface [20]. When $\nu$ corresponds to the EP i.e. $\nu=\nu^{*}$, it can be noticed that the two eigenvalues are equals. The case of a real-valued parameter $\nu \in \mathbb{R}$, like outline in Fig. 4(b) for $k_{3}=3$, is equivalent to a slice of the Riemann surface associated to the eigenvalues and is conveniently plotted in Fig. 5 .

Analytical details are postponed to the next section, but it can be observed in Fig. 5 that the defective eigenvalue will split into two simple eigenvalues as soon as the parameter is detuned from the EP. The EP is a branch point singularity and the local behavior of the merging pair of eigenvalues depends on the square root of the distance between the parameter and the EP. This distance gives also the radius of convergence $\rho$ of the Taylor expansion of the merging eigenvalues

$$
\rho\left(T_{\lambda_{i}}\right)=\left|\nu_{0}-\nu^{*}\right|
$$

In this case, $\rho \approx 0.6 \mathrm{~N} \mathrm{~m}^{-1}$ which is consistent with the results presented in Fig. 3 . In conclusion, the more the EP will be close to the real axis $\left(\mathfrak{I m}\left(\nu^{*}\right) \rightarrow 0\right)$, the more the veering phenomenon will be exacerbated and the radius of convergence of the Taylor series will be limited.

\subsection{Estimation of the radius of convergence of Taylor expansion}

To efficiently use Taylor approximation, the knowledge of the radius of convergence is of primary interest i) to ensure the best use of the computational resources avoiding to compute higher order terms and ii) to avoid wrong prediction. Different criteria have been proposed to quantify the veering phenomenon [33, 34] which could help to characterize the validity domain of Taylor expansions. Nevertheless, estimating the radius of convergence of the Taylor series seems to be a more robust strategy and becomes possible when high order derivative are available. Two estimators of the radius of convergence can be used. The first is based on the roots $z_{n}(n=1, \ldots, N)$ of the Taylor expansion $T_{\lambda_{i}}$. Eigenvalues's Taylor polynomial expansion of order $N$ has $N$ complex roots (counting their multiplicities) in the parameter space. Moreover, it can be shown that the roots of high order polynomials tends to be located on a circle [43]. For this class of problem, the eigenvalues are usually non vanishing functions. The radius of this circle thus provides an upper bounds of the radius of convergence of the Taylor expansion because the reconstructed function is not supposed to vanish here. Here, the estimator is obtained as the average distance between the roots $z_{n}$ 


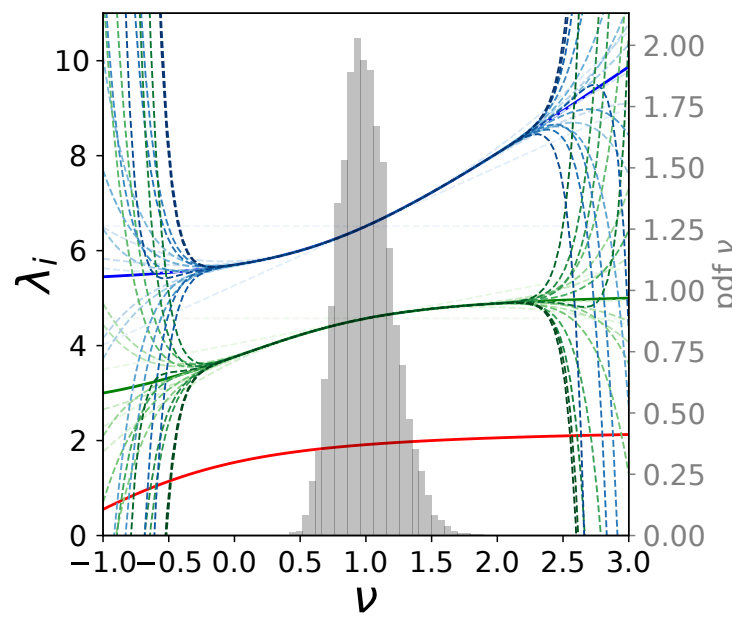

(a) Low veering, $k_{3}=4, \nu^{*}=0.6341+1.0323 \mathrm{i}$

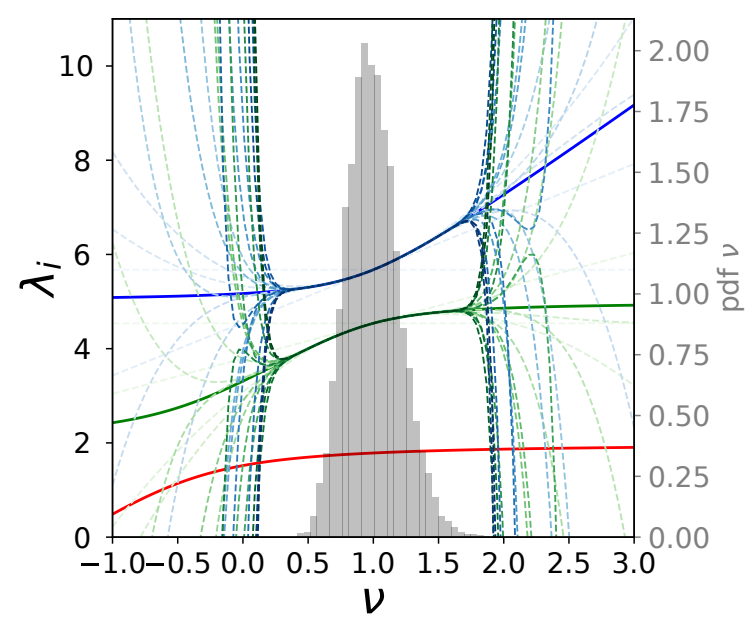

(b) Mild veering, $k_{3}=3, \nu^{*} \approx 0.8926+0.5977 \mathrm{i}$

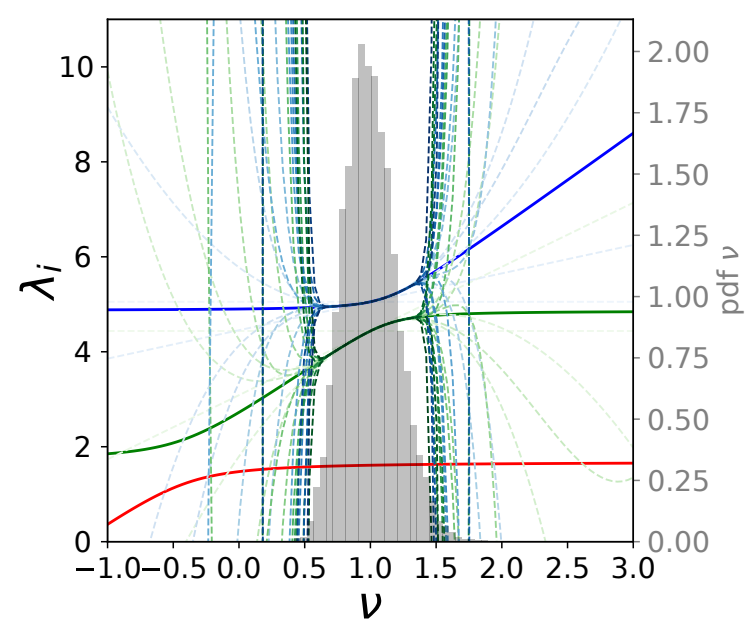

(c) Strong veering, $k_{3}=2.1, \nu^{*} \approx 1.147+0.2600 \mathrm{i}$

Figure 4: Impact of the veering phenomenon on the Taylor series approximation (dashed lines) for different approximation orders $(n \in \llbracket 1,20 \rrbracket)$ for the second and third eigenvalues of the 3 -Dof system at $\nu_{0}=1 \mathrm{~N} \mathrm{~m}^{-1}$. The pdf of the input random parameter is added for convinience. 


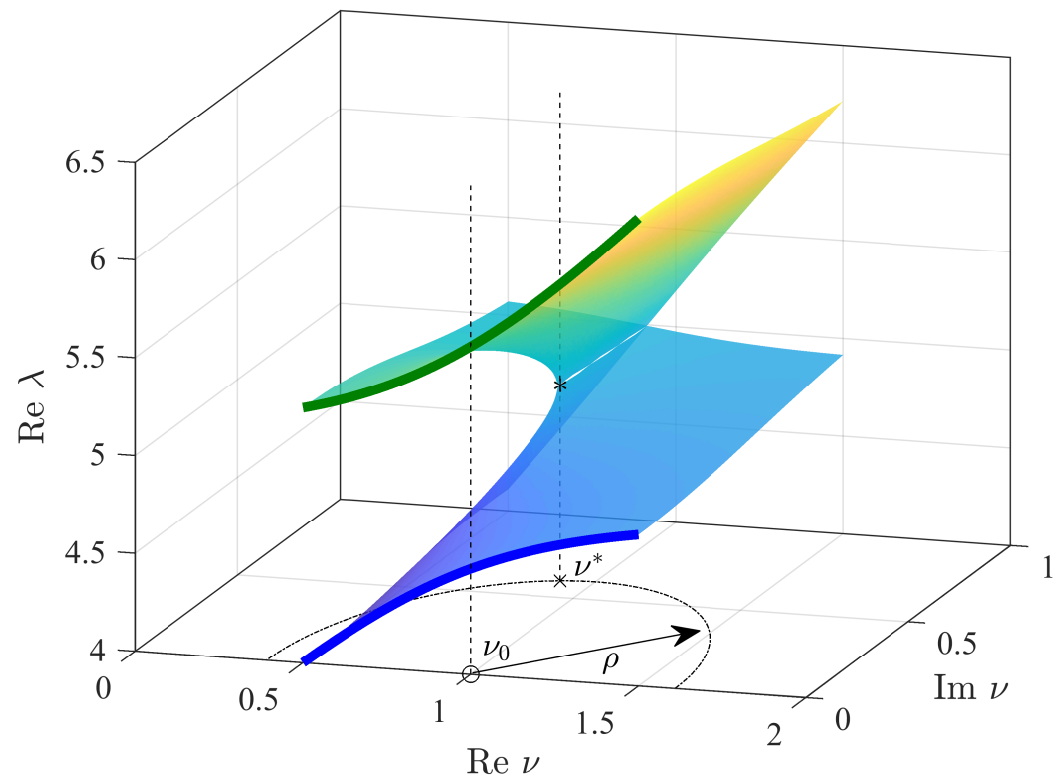

Figure 5: Riemann surface of the real parts of $\lambda_{2}$ and $\lambda_{3}$ for $k_{3}=3$ and illustration of the branch point singularity at the EP $\nu^{*}$.

of the Taylor series and the computation point $\nu_{0}$

$$
\rho_{N}^{\text {roots }}\left(T_{\lambda_{i}}\right)=\frac{1}{N^{\prime}} \sum_{n=1}^{N^{\prime}}\left|z_{n}-\nu_{0}\right| .
$$

For a sake of generality with other series representation used hereafter, $N^{\prime}$ denotes the number of spurious roots only, genuine roots have to be excluded.

Another estimator is based on the Cauchy-Hadamard's root test [41, sec. 2.6]. This criterion states that the radius of convergence at the point $\nu_{0}$ is defined as the superior limit when $n \rightarrow \infty$

$$
\rho_{N}^{\mathrm{CH}}\left(T_{\lambda_{i}}\right)=\left|t_{n}\right|^{-1 / N} .
$$

In practice, only the first terms of this sequence will be available, but hopefully, the truncated sequence can still provide a relevant convergence estimator.

Both estimators are compared in Tab. 1] and in Fig. 6 for the veering case presented in Fig. 5 . The exact EP locations are also presented as it provides the reference estimation of the radius of convergence. For strong veering case, ie $k_{3}=1.5$, it is shown in Tab. 1 that the radius depends clearly on the distance between the EP and the initial computational point $\nu_{0}$. When the real part of the parameter is very close to the real part of the EP, the radius of convergence is restricted to the imaginary part of the EP. The bound obtained by the truncated Cauchy-Hadamard sequence $\rho_{N}^{\mathrm{CH}}\left(T_{\lambda_{i}}\right)$ is illustrated in Fig. 6p) for $N \in \llbracket 1,22 \rrbracket$. This criterion is slightly tighter than those provided by $\rho_{N}^{\text {roots }}\left(T_{\lambda_{i}}\right)$. On the other hand, the root-based estimator oscillates less because of the averaging on several roots. It should be noticed that both estimators overestimate the radius of convergence even when the number of derivatives increases but remain cheap in terms of computational resources.

Recently an algorithm to precisely locate EP has been proposed by Nennig and Perrey-Debain [36]. It exploits high order eigenvalue derivatives and analytic continuation of the parameter in the vicinity of the EP. As EP provides the tightest bounds of the radius of convergence of Taylor series, this strategy will be investigated in the next section. 


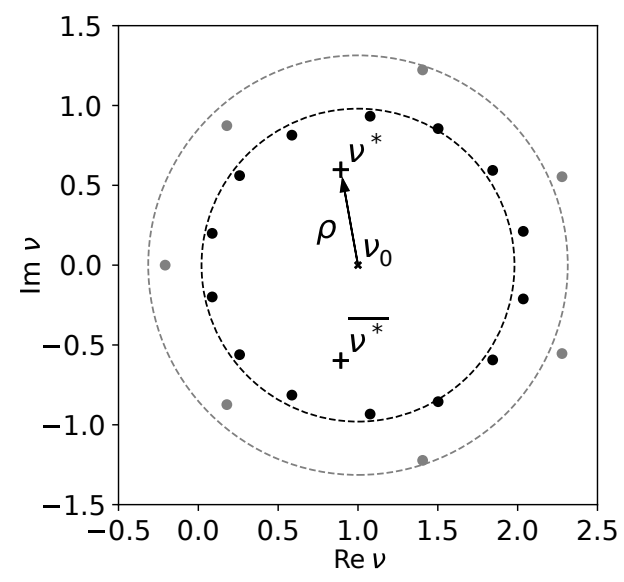

(a)

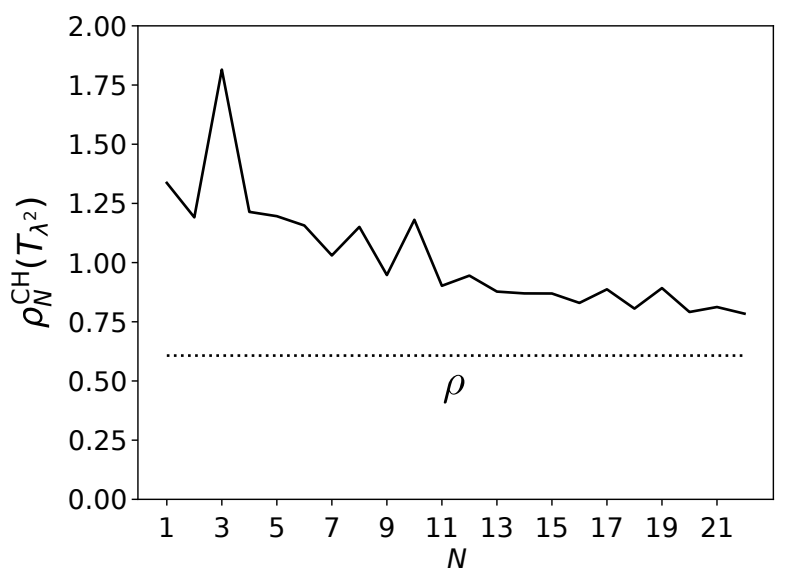

(b)

Figure 6: Illustration of a singularity of the complex eigenvalue loci and their impact on the radius of convergence $\rho$ of the Taylor series $\left(k_{3}=3\right)$. a) Roots of $T_{\lambda_{2}}(\bullet)$ for $N=8$ (gray) and $N=15$ (black) computed from $\nu_{0}=1$, the dotted line is a guide for the eye and is the mean radius of the roots distribution from Eq. 99; b) Cauchy-Hadamard's root test, the dotted line stand for the distance to the EP from Eq. 87.

Table 1: Estimators of the radius of convergence of the Taylor series of $\lambda_{2}$. The values are expressed in $\mathrm{Nm}^{-1}$.

\begin{tabular}{|c|c|c|c|c|c|c|c|c|c|}
\hline$k_{3}$ & $\nu_{0}$ & $\nu^{*}(\mathrm{EP})$ & $\rho\left(T_{\lambda_{2}}\right)$ & \multicolumn{3}{|c|}{$\rho_{n}^{\mathrm{CH}}\left(T_{\lambda_{2}}\right)$} & \multicolumn{3}{|c|}{$\rho_{n}^{\text {roots }}\left(T_{\lambda_{2}}\right)$} \\
\hline & & & & $N=5$ & $N=10$ & $N=15$ & $N=5$ & $N=10$ & $N=15$ \\
\hline 4. & 1. & $0.6341+1.0323 \mathrm{i}$ & 1.09 & 2.06 & 1.82 & 1.65 & 3.03 & 2.32 & 2.06 \\
\hline 3. & 1. & $0.8926+0.5977 \mathrm{i}$ & 0.61 & 1.21 & 0.95 & 0.87 & 1.80 & 1.13 & 0.98 \\
\hline 1.5 & 1. & $1.2618+0.1219 \mathrm{i}$ & 0.29 & 0.77 & 0.64 & 0.44 & 1.14 & 0.79 & 0.49 \\
\hline 1.5 & 1.26 & $1.2618+0.1219 \mathrm{i}$ & 0.12 & 0.35 & 0.28 & 0.19 & 0.52 & 0.41 & 0.21 \\
\hline
\end{tabular}




\section{Eigenvalues analytic continuation}

As illustrated in sec. 3.2 the veering phenomena becomes clearer when the parameter is allowed to be complex-valued. When the defective double root split, both eigenvalue exhibit a local square root behavior as shown in Fig. 5. Such behavior cannot be approached by a Taylor series, but is well captured by Puiseux series [20, 26.

Such series are considered as a generalization of Laurent series with fractional exponents of the function's indeterminate. They are widely used to represent locally algebraic surfaces such as the surface defined by the characteristic polynomial $D(\lambda, \nu) \equiv \operatorname{det}(\mathbf{K}(\nu)-\lambda \mathbf{M})=0$, with analytical dependency of $\nu$. As the radii of convergence of the Puiseux expansion and of the Taylor series are not bounded by the same kind of singularities in the complex-plane, the Puiseux expansion thus appears as a suitable approximation framework to extend perturbations methods to larger variability of the input parameters. A similar strategy has been previously proposed for mechanical applications by Luongo [4] to approximate eigenvalues with veering. However, the approach proposed by Luongo has lacked a generic and robust method to localize the EP and to compute the high order Puiseux series terms. The comparison between Puiseux and Taylor approximation and their combination will be discussed section 5 .

\subsection{Puiseux series}

Let us consider the Puiseux expansion of the merging pair of eigenvalues $\left(\lambda_{+}, \lambda_{-}\right)$about the point $\nu^{*}$ [20]

$$
\begin{aligned}
& P_{\lambda_{+}}=a_{0}+a_{1}\left(\nu-\nu^{*}\right)^{\frac{1}{2}}+\sum_{k=2}^{\infty} a_{k}\left(\left(\nu-\nu^{*}\right)^{\frac{1}{2}}\right)^{k}, \\
& P_{\lambda_{-}}=a_{0}-a_{1}\left(\nu-\nu^{*}\right)^{\frac{1}{2}}+\sum_{k=2}^{\infty} a_{k}\left(-\left(\nu-\nu^{*}\right)^{\frac{1}{2}}\right)^{k},
\end{aligned}
$$

although it could be generalized to an arbitrary number of modal merging. Here, both eigenvalues of the pair are described by the same Puiseux series up to a sign that defines the two branches of the Riemann surface that merge at the EP $\nu^{*}$ as shown in Fig. 5. As a consequence, for a given path in the complex parametric space, one can continuously change from one branch to the other. For instance, when $\nu$ move along the real axis, it explains the eigenvector switching at veering point [31. Puiseux series are the only representation that allow capture the Riemann surface topology and to follow each modal branch.

This kind of spectral degeneracy does not exist for real symmetric eigenvalue problems [31, 42]. Indeed, an Hermitian problem with two varying parameters can admit singularity referred to as Diabolic point [45] which corresponds to a conical intersection of the eigenvalue loci surface. For real non-symmetric problems, $\mathrm{EP}$ can be also found and are strongly related to stability issues [28, 29].

\subsection{Puiseux series coefficient computation}

The algorithm proposed by Nennig and Perrey-Debain [36] also exploits the high order derivatives of the veering pair of eigenvalues. The key idea is to introduced two analytic auxiliary functions of the eigenvalues pair

$$
\begin{aligned}
& g(\nu)=\lambda_{+}+\lambda_{-}, \\
& h(\nu)=\left(\lambda_{+}-\lambda_{-}\right)^{2},
\end{aligned}
$$

to remove the square root singularity. The method has 2 steps :

1. Find the EPs location by finding $h(\nu)$ roots $(h(\nu)$ vanishes when the two eigenvalues merge),

2. Get the coefficient $a_{0}, \ldots, a_{2 N}$ of the truncated Puiseux series by matching the truncated Taylor expansion of $T_{h}$ and $T_{g}$ with their expansion using the Puiseux series $P_{\lambda_{+}}$and $P_{\lambda_{-}}$. 
From the Puiseux series given in Eq. (11), it can be shown that $g(\nu)$ and $h(\nu)$ are analytic function so it is assumed they have convergent power series expansion around $\nu_{0}$

$$
\begin{aligned}
& T_{g}(\nu)=\sum_{n=0}^{N} b_{n}\left(\nu-\nu_{0}\right)^{n}, \quad \text { where } b_{n}=\left.\frac{g^{(n)}(\nu)}{n !}\right|_{\nu=\nu_{0}}, \\
& T_{h}(\nu)=\sum_{n=0}^{N} c_{n}\left(\nu-\nu_{0}\right)^{n}, \quad \text { where } c_{n}=\left.\frac{h^{(n)}(\nu)}{n !}\right|_{\nu=\nu_{0}} .
\end{aligned}
$$

Note that the smoothness of these functions has already been remarked in [46] 48] for $h(\nu)$ and in [20, p. 66] for $g(\nu)$.

The derivative of $g$ and $h$ at $\nu_{0}$ can be directly obtained from the derivative of the selected pair of eigenvalues

$$
\begin{aligned}
g^{(n)}\left(\nu_{0}\right) & =\lambda_{+}^{(n)}\left(\nu_{0}\right)+\lambda_{-}^{(n)}\left(\nu_{0}\right), \\
h^{(n)}\left(\nu_{0}\right) & =\sum_{k=0}^{\left\lfloor\frac{n}{2}\right\rfloor}\left(\begin{array}{l}
n \\
k
\end{array}\right)\left(2-\delta_{\frac{n}{2} k}\right)\left(\lambda_{+}^{(n-k)}\left(\nu_{0}\right) \lambda_{+}^{(k)}\left(\nu_{0}\right)+\lambda_{-}^{(n-k)}\left(\nu_{0}\right) \lambda_{-}^{(k)}\left(\nu_{0}\right)\right)-2 \sum_{k=0}^{n}\left(\begin{array}{l}
n \\
k
\end{array}\right) \lambda_{+}^{(n-k)}\left(\nu_{0}\right) \lambda_{-}^{(k)}\left(\nu_{0}\right),
\end{aligned}
$$

where upperscript ${ }^{(n)}$ denotes the $n^{\text {th }}$ derivative with respect to the complex parameter $\nu$. Here, $\delta_{\frac{n}{2} k}$ is the Kronecker symbol generalized to rational numbers and is equal to zero whenever $n$ is odd and symbol \lfloor\rfloor is the floor function. These convention have been introduced in order to take into account symmetries in the expression to optimize the computation speed.

Since $h(\nu)$ is known as a Taylor polynomial, roots of $T_{h}(\nu)$ are obtained by companion matrix method. As already discussed for $T_{\lambda_{i}}$, the spurious roots of a Taylor polynomial tends to be aligned on a circle. Genuine zeros, which therefore corresponds to EP, can be located as they stand apart from the circle boundary [43. The second step is to get Puiseux series coeffcient $a_{k}$. The basic idea is to compute separately the even and the odd terms using the auxiliary functions $h$ and $g$. The $N$ first derivatives for each eigenvalue are exploited to compute the $2 N$ first terms of the Puiseux series at the EP. This part is detailed in the Appendix Appendix B. To get Puiseux series coefficients, other approaches exist, like those proposed in Refs. [28, 49] but they are limited to first or second terms and the computation performed at EP are hard to handle numerically. The advantage of the method proposed in [36] is to compute eigenpairs and derivatives far from the singularity where the standard eigenvalue solver may encounter some difficulties.

The roots of $T_{h}$ Taylor series computed at $\nu_{0}$ are given in Fig. 7. Comparing this figure to Fig. 6, it can be observed that the estimator of radius of convergence $\rho^{\text {roots }}\left(T_{h}\right)=2.7$ is larger than $\rho^{\text {roots }}\left(T_{\lambda_{2}}\right)=0.6$. As expected, the function $h$ is smoother than the eigenvalue $\lambda_{+}$and $\lambda_{-}$because the branch point singularity has been removed. Although this approach is efficient to remove all the singularity involving the two selected eigenvalues, it is not possible to remove singularities involving one of the eigenvalue from the pair and another eigenvalue of the system. The singularity involving the merging of $\left(\lambda_{1}, \lambda_{2}\right)$ has also been found by the proposed algorithm at $\nu_{12}^{*}=-0.4482 \pm 0.8305 \mathrm{i}$. This second EP limits the radius of convergence of $T_{h}\left(\lambda_{2}, \lambda_{3}\right)$. In Fig. $\left.7 \mathrm{~b}\right)$, the computation point $\nu_{0}$ is moved further away from the EP $\nu_{12}^{*}$, the radius of convergence is clearly larger but is still bounded by the EP $\nu_{12}^{*}$. The radius of convergence of $T_{g}$ has exactly the same limitation.

\subsection{Radius of convergence of Puiseux series}

It has been shown in the previous sections which kind of singularity may limits the radius of convergence of eigenvalues Taylor expansion $T_{\lambda_{i}}$ and the analytic auxiliary function $T_{h}$ or $T_{g}$. It remains to state the convergence limits of the Puiseux expansion given by Eq. (11). Puiseux series $P_{\lambda_{i}}$ provides a local description of the eigenvalue locus in the vicinity of the EP. However, according to the implicit function theorem, the Puiseux local representation of the algebraic surface $D(\lambda, \nu)=0$, ceases to converge when $\frac{\partial D(\lambda, \nu)}{\partial \lambda}=0$. This double root condition of the characteristic polynomial $D(\lambda, \nu)$ is equivalent to an EP. Thus, to characterize, a priori, the radius of convergence of the Puiseux series, a criterion can be defined by the distance between the 


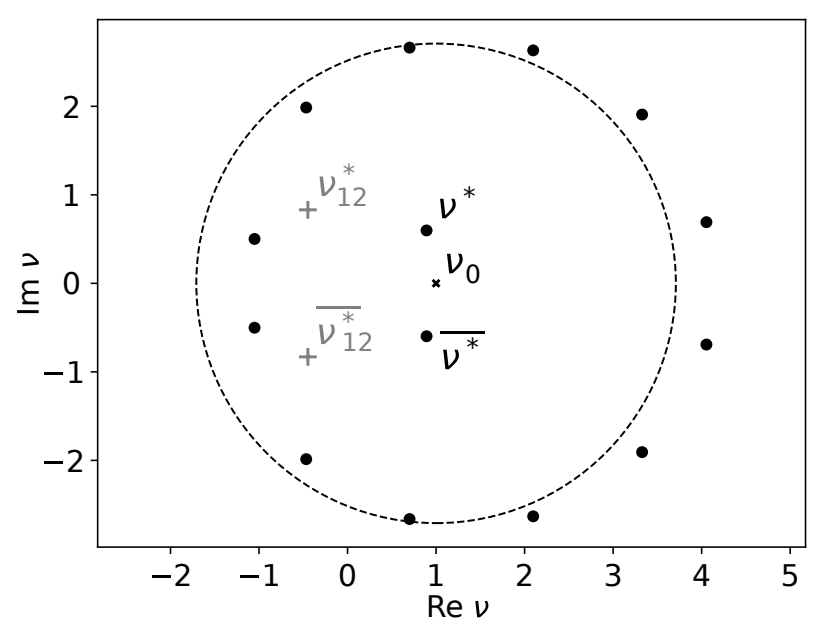

(a) $\nu_{0}=1$.

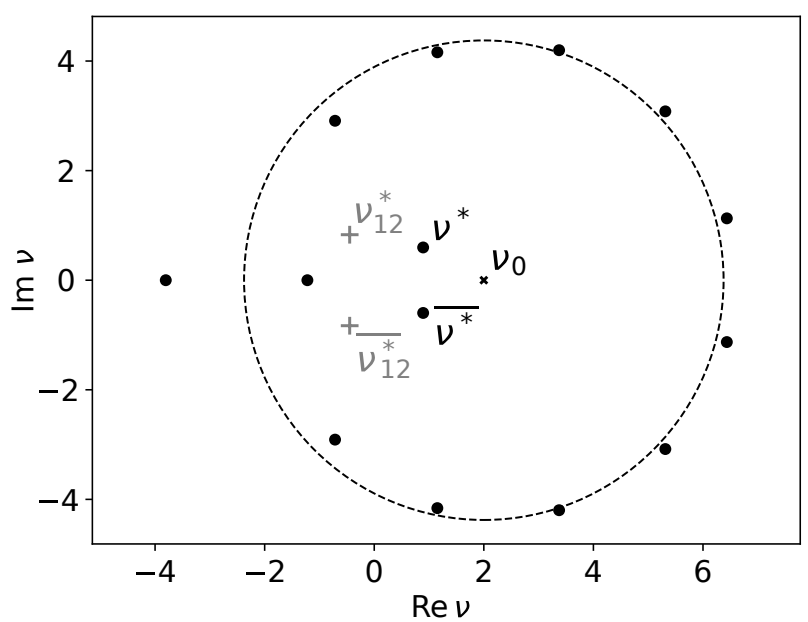

(b) $\nu_{0}=2$.

Figure 7: Roots of Taylor expansion of $T_{h}\left(\lambda_{2}, \lambda_{3}\right)$ and EP location when $k_{3}=3$ and $N=15$. The $\bullet$-markers stand for the roots of $T_{h}$ and + markers indicate EPs of other eigenvalues pairs, namely $\nu_{12}^{*}$ and $\bar{\nu}_{12}^{*}$. a) For $\nu_{0}=1, T_{h}$ exact radius of converge is $\left|\nu_{0}-\nu_{12}^{*}\right|=1.66$ and its estimation is $\rho^{\text {roots }}\left(T_{h}\right)=2.7$; b) For $\nu_{0}=2$, further away from the EP $\nu_{12}, T_{h}$ exact radius of converge is $\left|\nu_{0}-\nu_{12}^{*}\right|=2.58$ and its estimation is $\rho^{\operatorname{roots}}\left(T_{h}\right)=4.4$.

EP used to compute the Puiseux series to the closest other EP. Actually, it corresponds to the EP associated to the same pair of eigenvalues but for the complex conjugate value of the parameter. It can be easily shown from Eq. (1) that the complex conjugated value of the parameter yields to a complex conjugated eigenvalue if $\nu$ is only one complex variable in the problem. These EPs pairs can be observed in Figs. 6(a) and 7 .

Let us note $\nu^{*}=a+\mathrm{i} b$ the point where the Puiseaux series are computed and $\overline{\nu^{*}}=a-\mathrm{i} b$ the complex conjugate value of the EP. It follows that the radius of convergence of the puiseux expansion in the complexplane do not exceeding $2 b$. It can be noticed that it is twice the radius of convergence of the Taylor expansion when computed at $\nu_{0}=a$. If the parameter is real valued and $\nu_{0}=a$, the Taylor series validity domain is then limited to $[a-b, a+b]$, while the Puiseux series validity domain is $[a-b \sqrt{3}, a+b \sqrt{3}]$, the effective gain is therefore $\sqrt{3}$. Numerical experiment is presented in Fig. 8 for weak and strong veering configurations when $\nu_{0} \approx a$. This figure presents the error isolines at $1 \%$ and the theoretical prediction of the convergence disk for the Taylor and the Puiseux approximations of $\lambda_{2}$. In both cases, the theoretical prediction of the convergence disk are in good agreements with the numerical simulation and slightly underestimate the radius of convergence. When the veering become stronger, both $\mathrm{EP} \nu^{*}$ and $\overline{\nu^{*}}$ move to the real axis and the radius of convergence of Puiseux series (and Taylor) decrease, but Puiseux series radius of convergence still remains $\sqrt{3}$ larger than Taylor series one.

The random eigenfrequencies of a structure could thus be characterized using the Puiseux approximation from realizations of the input parameter respectively within the intervals $\nu(\theta) \in[-0.1,1.9]$ and $\nu(\theta) \in$ $[1 ., 1.5]$ for the weak and strong veering configurations. For these configurations, assuming the input random parameter to be a random variable centered on $\mu \approx a$, the largest variability which can be handled with the Puiseux approximation can be defined with a simple two-sigma criterion. This way, $95 \%$ of the realizations of the parameter will lie within the interval $[a-b \sqrt{3}, a+b \sqrt{3}]$. With a classical Taylor approximation, this interval has to be reduced to $[a-b, a+b]$. Let us note $\sigma_{P}$ and $\sigma_{T}$ the maximum standard deviation of the random input parameter allowing to ensure a good approximation of the eigenvalues respectively with Puiseux and Taylor approximations. The two-sigma criterion implies that $4 \sigma_{P} \leq 2 b \sqrt{3}$ and $4 \sigma_{T} \leq 2 b$. When centered on the real part of an EP, the validity domain of Puiseux and Taylor approximations can be defined by the maximum coefficient of variation of the input random parameter ensuring the two-sigma criterion. The relation between the maximum coefficient of variation admissible for Puiseux and Taylor approximations is therefore

$$
\delta_{P_{\lambda_{i}}}=\sqrt{3} \delta_{T_{\lambda_{i}}} .
$$

The last point to investigate concerns the validity of the computed Puiseux series coefficients. It is 


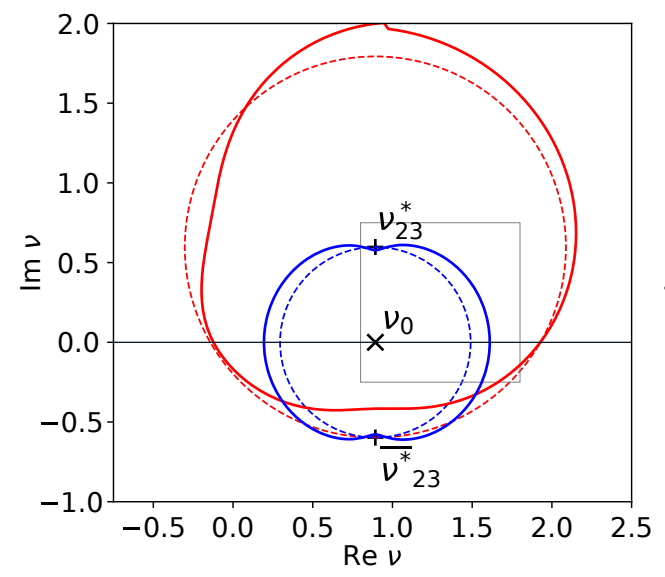

(a)

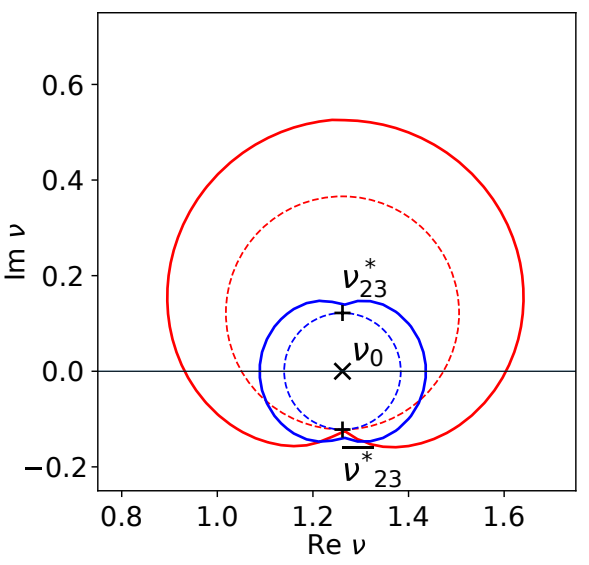

(b)

Figure 8: Reconstruction error isolines at $1 \%$ (solid line) in the $\nu$-complex plane with $\nu_{0} \approx a\left(\right.$ red: $P_{\lambda_{2}}$, blue: $T_{\lambda_{2}}$ ) and the theoretical radii of convergence (dash line). a) Weak veering $\nu^{*}=1.262+0.122 j, k_{3}=3$, b) Strong veering $\nu^{*}=0.893+0.598 j$, $k_{3}=1.5$ with a smaller radius of convergence. The gray frame aims to help the scale comparison between the two subfigures.

noteworthy that the presence of this complex conjugated EPs pair does not affect the analyticity of $h$ and $g$ because all shared singularity in the pair are removed from the analytic auxiliary functions [31, 36]. Thus EP and Puiseux series coefficient can be obtained as long as the EP belong to the convergence disk of the Taylor series $T_{h}$ and $T_{g}$. This domain is generally larger than the Puiseux series convergence disk. It means that, once an EP has been located from $\nu_{0}$ and the Puiseux series coefficients have been obtained, this series can be uses in the vicinity of the EP even if the $\nu_{0}$ is outside the radius of convergence of the Puiseux series. This remarks is important because the same computation can be exploited through a Taylor series expansion in the vicinity of $\nu_{0}$ and the Puiseux series can be use when Taylor break down in the vicinity of the $\mathrm{EP} \nu^{*}$.

\subsection{Eigenvalue reconstruction from analytic auxiliary function}

The discussion initiated in the previous section on the operable domain to compute the Puiseux series coefficents has highlight the good properties of the analytic auxiliary function $h$ and $g$. It has been shown that the convergence domain of the Taylor series $T_{h}$ and $T_{g}$, is generally larger than the Puiseux series convergence disk. As a consequence, we propose to exploit these functions to directly reconstruct the pair of eigenvalues on a wide range of the varying parameter. From the definitions of $g$ and $h$ given in Eqs. (12) and (13), the two eigenvalues can be written as a function of $g$ and $h$

$$
\begin{aligned}
& \lambda_{+}=\frac{g+\sqrt{h}}{2}, \\
& \lambda_{-}=\frac{g-\sqrt{h}}{2} .
\end{aligned}
$$

These expressions can be approximated through the truncated Taylor series $T_{h}$ and $T_{g}$

$$
\begin{aligned}
& A_{\lambda_{+}}=\frac{T_{g}+\sqrt{T_{h}}}{2}, \\
& A_{\lambda_{-}}=\frac{T_{g}-\sqrt{T_{h}}}{2} .
\end{aligned}
$$

This new representation allows to bypass the singularity at $\overline{\nu^{*}}$ and to considerably increase the validity range of the reconstruction of the eigenvalues pair.

A comparison between the approximation based on analytic auxiliary function $A_{\lambda_{ \pm}}$and a direct eigenvalue computation is provided in Fig. 9 for the eigenvalues pair $\left(\lambda_{2}, \lambda_{3}\right)$. The $1 \%$-error isolines of $\left(\lambda_{2}, \lambda_{3}\right)$ 


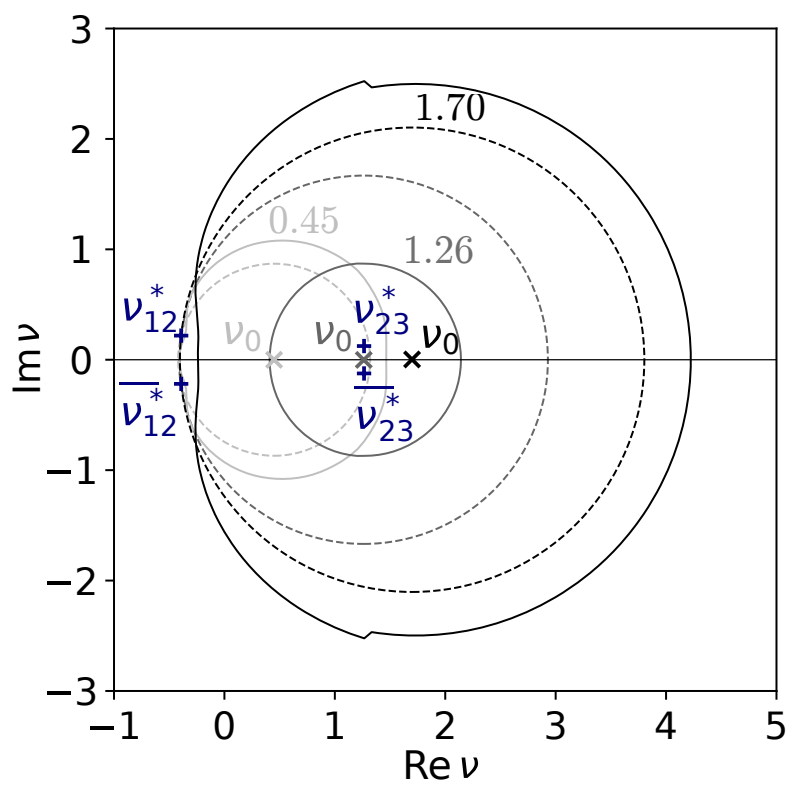

(a) $N=15$

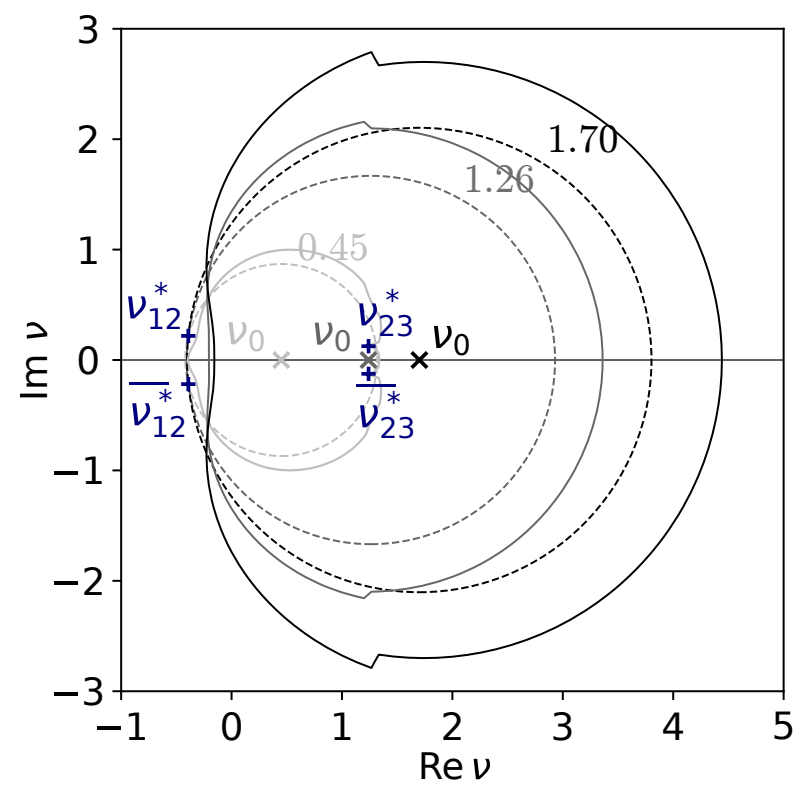

(b) $N=10$

Figure 9: Error isolines at $1 \%$ for analytic auxiliary function reconstruction (solid lines) for $\nu_{0} \in\{0.45,1.26,1.70\}$ and comparison with the theoritical convergence bounds (dashed lines) for strong veering $k_{3}=1.5$.

for a strong veering case $k_{3}=1.5$ are given for three computation points $\nu_{0}$ such as : i) $\nu_{0}$ is at equal distance of $\nu_{12}^{*}$ and $\nu_{23}^{*}$; ii) $\nu_{0}=\mathfrak{R e} \nu_{23}^{*}$, and iii) $\nu_{0} \gg \mathfrak{R e} \nu_{23}^{*}$. When $N=15$, it can be observed in Fig. 9(a) that the radii of convergence of $T_{h}$ are limited by the presence of the EPs involving one eigenvalue from the pair and another one, i.e. $\nu_{12}^{*}$. The theoretical bounds are thus accurate and provide a good estimation of the convergence radius of the representation when the computational point $\nu_{0}$ is not too close from the EP $\nu^{*}$. For this latter case, the numerical experiment shows a suboptimal radius of convergence. This can be explained by the coefficients of the Taylor series $T_{h}$ which increase dramatically. Limiting $N=10$, where $\left|c_{10}\right| \approx 1000$ solve the problem, and the theoretical bound is recovered as shown in Fig. 9b). In the neighborhood of the EP, although $h$ and $g$ are analytic, their Taylor series is based on the eigenvalue derivatives which are unbounded because of the EP singularity. As a consequence, coefficients of the Taylor series may be prone to round off error for strong veering case (e.g. $\left.k_{3}=1.5\right)$. A more robust approach is to truncate the series if its coefficients become too high.

Finally when, $\mathfrak{R e} \nu_{0} \gg \mathfrak{R e} \nu^{*}$, because this $3 \mathrm{DoF}$ system as only 3 eigenvalue branches, there is no other EP on the right side of $\nu_{0}$. This explain the huge validity range of the reconstruction $A_{\lambda_{i}}$ which is bounded only on the left side by $\nu_{12}^{*}$.

\section{Comparison of the proposed approaches}

Computing high order derivatives allows to switch between several representations to reconstruct the eigenvalue loci. This section summarizes and compares each representation in term of convergence and validity range and ends by the proposition of an algorithm formalizing the more suitable approach to efficiently approximate the eigenvalue loci with real input parameter.

Let us consider a stringent test case with strong veering between $\lambda_{2}$ and $\lambda_{3}$ by tuning the stiffness parameter such $k_{3}=1.5$. Two computation points $\nu_{0}$, respectively outside and inside the veering area, are then studied to compare the strength and weakness of each approaches. 


\subsection{Computation point outside of a veering area}

Fig. 10(a) illustrates the eigenvalue loci and their different approximations using Taylor series, Puiseux series and the analytic auxiliary functions for real values of $\nu$, corresponding to the Hermitian eigenvalue problem (1). All representations are computed from the point $\nu_{0}=0.5$ outside of a veering area. It can be noticed that the Taylor approximation (solid lines) fits well the direct computation solution until it reaches the veering area. On the other hand, the Puiseux expansion (dash-dotted lines) fails in vicinity of $\nu_{0}$, because the computation point is too far from the EP, but accurately follows the reference computation in the veering area even if the computation point is outside of the Puiseux radius of convergence. Finally, the analytic auxiliary function reconstruction (dashed lines) combines benefits of both other approximations and well fits the eigenvalue loci for a wide range of $\nu$ including the veering area related to the pair $\left(\lambda_{2}, \lambda_{3}\right)$.

When $\nu$ spans the whole complex-plane, the eigenvalue problem become non-Hermitian, the results are given in Fig. 10(c) This figure presents the error isolines at $1 \%$ of the Taylor (blue), Puiseux (red) and analytic auxiliary function (black) approximations of $\lambda_{2}$ and $\lambda_{3}$ with respect to a direct computation of the eigenvalue problem (1). The theoretical radius of convergence of each approximation, bounded by the EPs positions, are also overprinted. In each cases, the theoretical prediction of the convergence disk is in good agreements with the numerical simulation and slightly underestimate the actual radius of convergence.

As expected from previous section, the Taylor approximation is centered on $\nu_{0}$ and bounded by the EP $\nu_{23}^{*}$ associated to the veering phenomenon between $\left(\lambda_{2}, \lambda_{3}\right)$. The validity domain of the Puiseux approximation is centered on the EP $\nu_{23}^{*}$ and is bounded by the complex conjugate EP $\bar{\nu}_{23}^{*}$. The auxiliary function reconstruction is centered on $\nu_{0}$ and bounded by the EP $\nu_{12}^{*}=-0.3918+0.2185 i$ corresponding to the merging of $\lambda_{2}$ and an other eigenvalue of the problem, here $\lambda_{1}$. It should be kept in mind that this theoretical radius is not known until applying the EP localization algorithm to the pair of eigenvalue $\left(\lambda_{1}, \lambda_{2}\right)$. However, it can be estimated using the estimator $\rho^{\text {roots }}\left(T_{h}\right)$ proposed in Eq. (9). In practice, merging involved contiguous eigenvalue thus if a group of $K$ eigenvalues $\lambda_{i},, \lambda_{i+K}$ have to be reconstructed, most of the EPs can be located and exact theoretical radius can be obtained for the analytic auxiliary function reconstruction approach excepted for those involving $\lambda_{i}$ and $\lambda_{i+K}$.

In order to characterize the random eigenfrequencies of the 3-DoF system, the analytic auxiliary function approximation can be used to generate the realizations of the random eigenfrequencies from the realizations of the input parameter within the intervales $\nu(\theta) \in[-.25,1.5]$ with one computation. Note that the negative part of this interval has no physical meaning for the stiffness of standard materials but may be usefull in the other context, e.g. for metamaterial design. Considering a simple two-sigma criterion as in section 4.3 and assuming a symmetric distribution of $\nu(\theta)$ centered on $\nu_{0}$, the coefficient of variation of the input random parameter $\nu(\theta)$ which can be handle by the proposed approximation without significant accuracy loss is about $\delta_{\nu}=\frac{\sigma_{\nu}}{\mathrm{E}[\nu]}=0.875$. Which is higher than variability classically handled by low order perturbation methods.

As a partial conclusion, far from the veering zone, the direct eigenvalue Taylor expansion $T_{\lambda_{i}}$ and the analytic auxiliary functions scheme behave well, but for robustness the analytic auxiliary functions should be preferred, because the a priori knowledge of the veering zone is difficult to know.

\subsection{Computation point in a veering area}

The second case compares the three proposed approximations computed at point $\nu_{0}=1$ which is close to the real part of the EP $\nu_{23}^{*}$ and therefore inside a veering area of $\left(\lambda_{2}, \lambda_{3}\right)$. Fig. 10(b) illustrates the eigenvalue loci and the different representation for real values of $\nu$. It can be noticed that the Taylor and Puiseux approximations (respectively solid lines and dash-dotted lines) are limited to a small domain in the veering zone since $\mathfrak{I m} \nu^{*} \rightarrow 0$. On the other hand, the analytic auxiliary function representation well fits the direct computation on a wide range of $\nu$ including the veering area. Fig. 10(d) presents the error isolines at $1 \%$ of the Taylor (blue), Puiseux (red) and analytic auxiliary function (black) approximations of $\lambda_{2}$ and $\lambda_{3}$ when $\nu$ varies in the complex-plane. This error is computed using direct eigenvalue computation as a reference. It can be noticed that the theoretical radius of convergence of the reconstruction based on analytic auxiliary function remains centered on $\nu_{0}$ and bounded by the EP $\nu_{12}^{*}$ between $\lambda_{2}$ and $\lambda_{1}$. Here, its radius of convergence is larger than in the previous case, illustrated in Fig. 10(a) because $\nu_{0}$ is farther from $\nu_{12}^{*}$. This approximation thus provides an accurate representation of the eigenvalues pair $\left(\lambda_{2}, \lambda_{3}\right)$ for $\nu \in[-0.25,2.25]$. Thus, the analytic auxiliary functions approximation allows to consider an input random 


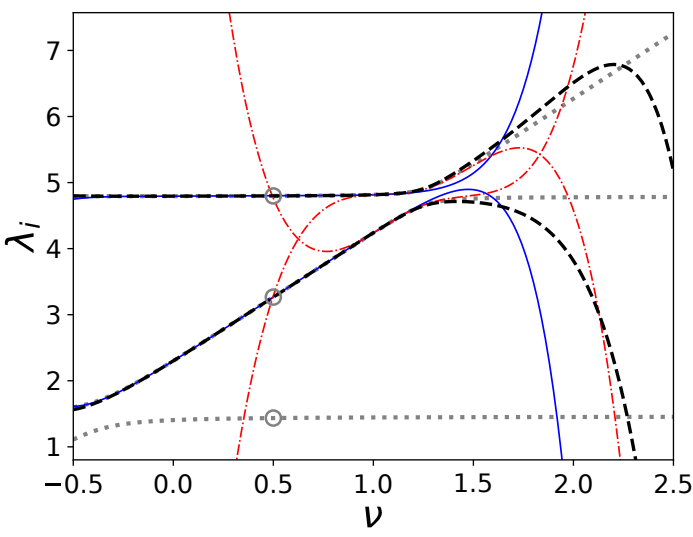

(a)

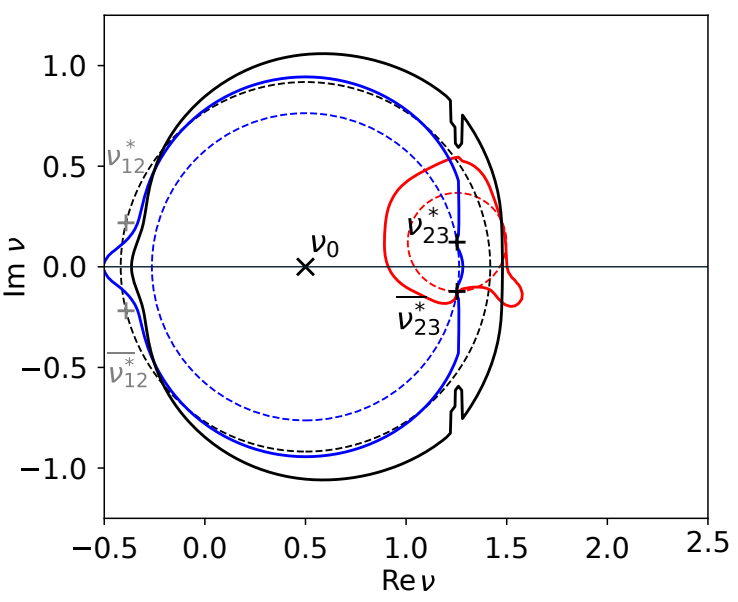

(c)

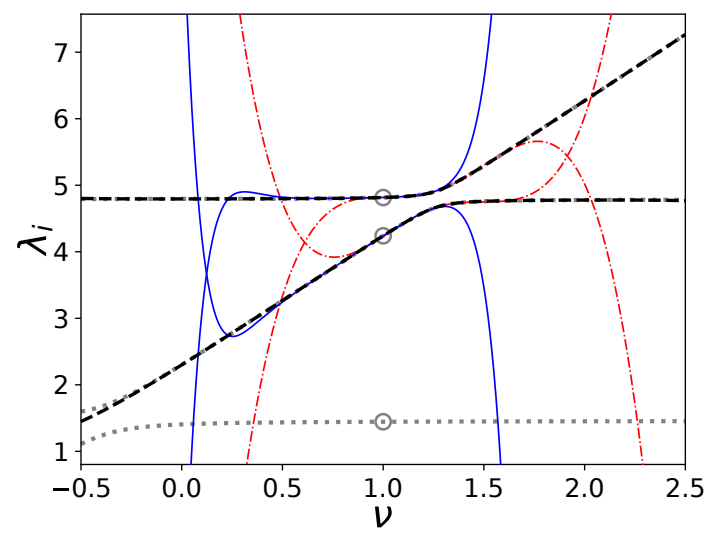

(b)

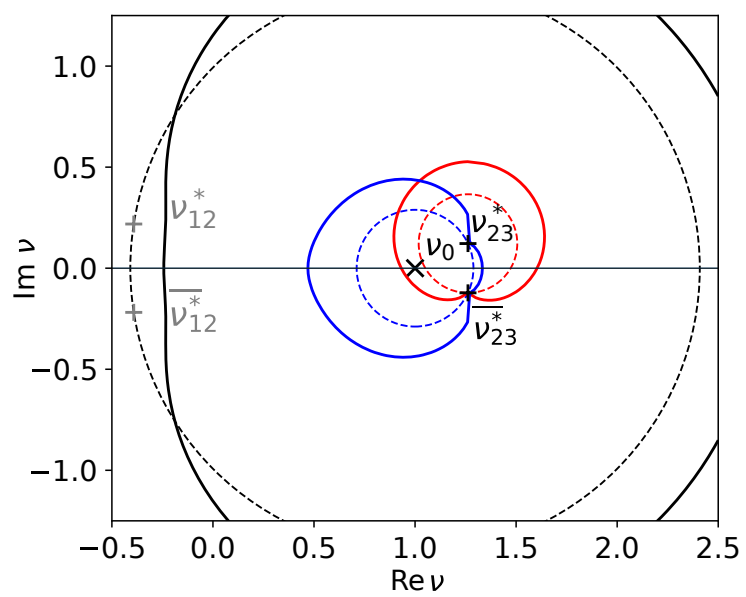

(d)

Figure 10: Comparison of eigenvalue loci reconstructions with Taylor (blue), Puiseux (red) and Analytic auxiliary functions (black) approximations for strong veering configuration $k_{3}=1.5$. When $\nu$ spans the real axis with $\nu_{0}=0.5(\mathrm{a})$ and $\nu_{0}=1.0$ (b). When $\nu$ spans the complex plane, reconstruction error isolines at $1 \%$ for the different approximations when computation point is $\nu_{0}=0.5$ (c) and $\nu_{0}=1.0$ (d). The dash line denotes theoretical converge radius. In all cases $N=10$.

variable centered on $\nu_{0}$ with coefficient of variation equal to $\delta_{\nu}=0.625$ to characterize the pair of considered random eigenfrequencies.

To conclude, close to the veering zone, the analytic auxiliary functions scheme should be preferred if the complex conjugate of the EP is present.

\subsection{Approximations overview}

The pro and cons of each tested representation are conveniently summarized here:

Eigenvalue Taylor expansion around $\nu_{0}, T_{\lambda_{i}}$ : This is the most common approach, which is efficient as long as the veering is weak because its radius of convergence is limited by the presence of the EP. Using higher order expansion may be computationally efficient if direct method 39] are used to compute the derivative of few selected eigenvalues. This approach can be easily extended to multivariate case.

Eigenvalue Puiseux expansion around the EP $\nu^{*}, P_{\lambda_{i}}$ : This approach exploits the local behavior in the vincinity of the EP and is the only one able to reconstruct the good branch structure of the eigenvalue Riemann surfaces which is crucial for tracking branches. The convergence of $P_{\lambda_{i}}$ is limited by the presence of additional EP involving $\left(\lambda_{+}, \lambda_{-}\right)$like $\overline{\nu^{*}}$ in the tested example. This limitation 
mainly concerns Hermitian problems with strong veering but leads to good reconstruction when EP are well separated as in 36.

Analytic auxiliary functions Taylor expansion around $\nu_{0}, A_{\lambda_{i}}$ : This approach is the more robust and its radius of convergence is limited by the presence of the EPs involving one eigenvalue from the pair $\left(\lambda_{+}, \lambda_{-}\right)$and another one. If there is no veering, validity range will be similar to $T_{\lambda_{i}}$, but when veering is visible, this approach is recommended. It seems possible to extend it to multivariate case as long as the EP are isolated.

\subsection{Proposed algorithm}

Based on the preceding results, this section presents a general algorithm to efficiently reconstruct the eigenvalue loci using the analytic auxiliary function approximation from a local computation at $\nu_{0}$. This algorithm, detailed in Algo. 1, can be used to generate the realizations of the random eigenfrequencies of the structure and to characterize their pdf. When compared with standard perturbation methods, the main advantages of the proposed approach are a larger validity domain and an estimation a priori of the radius of convergence which allows to make the best use of available computational resources. These enhancements are possible by removing the EP branch point singularity by considering a pair of eigenvalues simultaneously.

This allows to significantly reduce the computational cost in comparison with a Monte-Carlo simulation considering the entire structure model. This algorithm could also be particularly relevant for many other applications based on parametric eigenvalue problems (rotating machinery, waveguide attenuation, instability problems as squeal or flutter).

The input data of this algorithm are the two first statistical moments of the random input parameter, the operator associated to the eigenvalue problem and its derivatives with respect to the input parameter $\nu$, a safety factor $\xi$ and a tolerance tol. The statistical moments of the input parameter allows to estimate a priori the required validity domain of the eigenvalue approximation.

For a given pair of eigenvalues, the analytic auxiliary function representation is bounded by the existence of EPs relative to an eigenvalue of the pair and an other eigenvalue of the problem. However, these bounding EPs cannot be located precisely without computing the $\mathrm{n}^{\text {th }}$ derivatives of the closest eigenvalues of the considered eigenvalues pair. A cheaper solution is to estimate the radius of convergence of $T_{h}$ and $T_{g}$ using Eq. (9). As this estimator overestimates the convergence radius, we introduce the safety factor $\xi$. It has been shown from Tab. 1 that $\xi$ is about $\frac{1}{3}$ when the Taylor expansion order $N$ is between $6-8$ and about $\frac{1}{2}$ when $N$ is between to $10-12$. As illustrated section 4.4 when the veering phenomenon is significant and the computation point is close to the real part of the EP, the reconstruction of the eigenvalue loci could be polluted by round off error. The tolerance tol allows to avoid this drawback by truncating the Taylor series when its coefficient exceed the tolerance value.

To monitor a pair of eigenvalues, the first step is to solve the generalized eigenvalue problem (1). Then, the eigenvalues derivatives are obtained by solving an augmented linear system for each eigenvalue. With a direct solver, the matrix is factorized once then forward and backward substitution are used to solve the linear system for multiple RHS. This approach is scalable and can be easily extended to sparse matrices encounter commonly in structural dynamics [36]. For big size problems, say $10^{5}$ to $10^{6}$ dofs, these steps represent almost the total computational time, because the computation of the RHS from Eq. (7) is generally faster. Indeed, for sparse matrices, the matrix-vector product present in the RHS scale as the number of non zeros of the matrix and remains fast compared to the matrix factorization. This last case has been tested in Ref. [36]. It has been shown that the equivalent time to compute the $10^{\text {th }}$ first derivatives of a pair of eigenvalues is equivalent to solve 2 to 4 the eigenvalue problems depending of the problem size.

In situations where the operator derivatives are not available, which is often the case when using with black-box FEM solvers, numerical differentiation using finite differences can be used. If the operator is a linear function of the parameter, only the first derivative is requiered in the recurence formula (7) and a non-intrusive version of the method can be achieve. Similar approach has been used for instance in [25].

The proposed approach has been implemented in a dedicated open source python library available at github.com/nennigb/EasterEig. The three dof system test case, guideline example of this paper, is provided in the example folder. 


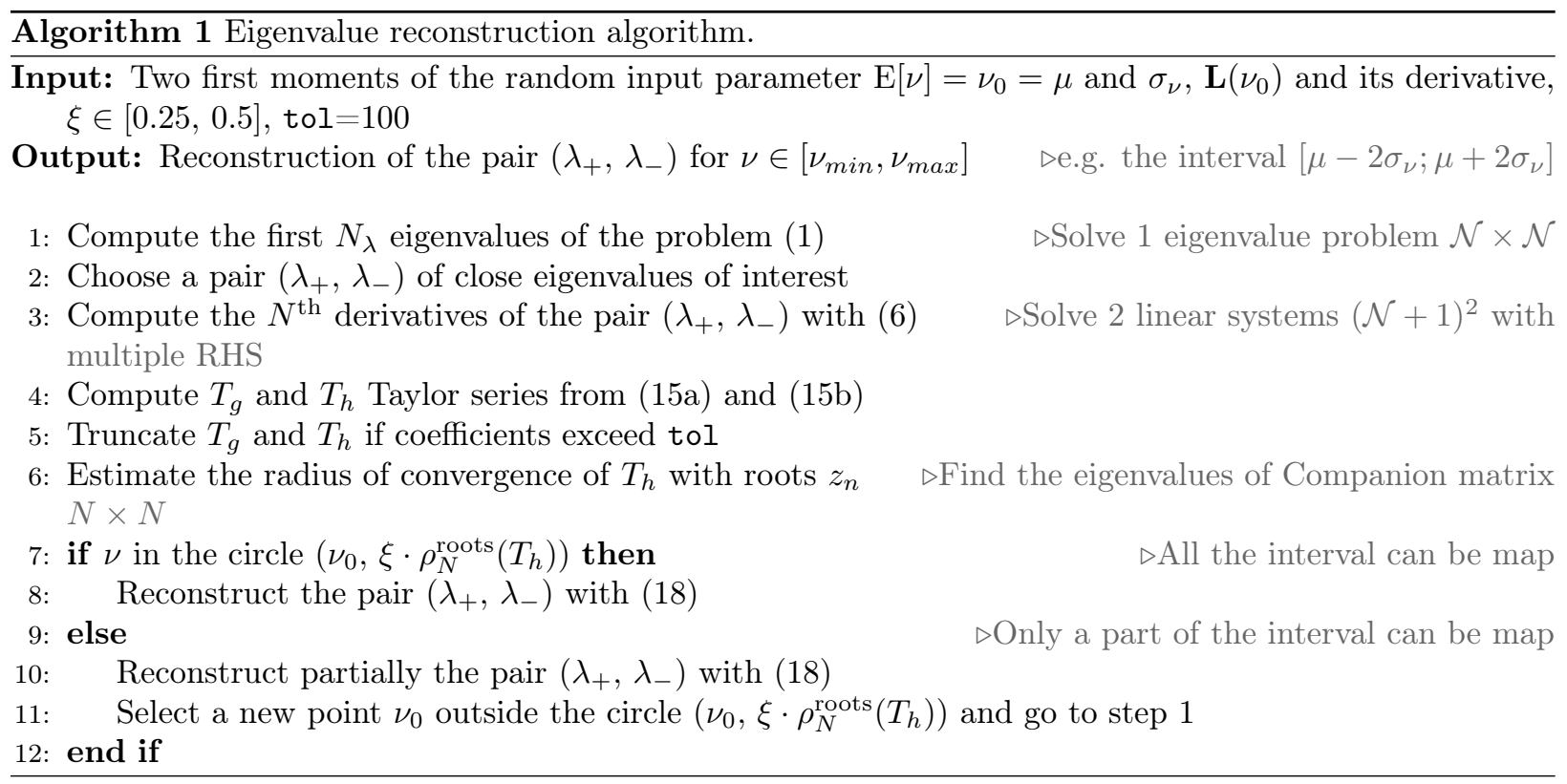

\section{Conclusion}

This paper presents a method to reconstruct the eigenvalue loci of a structure with a random parameter based on higher order derivative of the eigenvalues. Such derivatives are obtained using the bordered matrix formalism [39] and allow i) to switch between several representations of the eigenvalues to get analytic continuation; ii) to estimate the radius of convergence of each eigenvalue representation.

The difficulties encountered in the eigenvalue reconstruction are closely related to the existence of a branch point singularities in the parameter complex-plane referred to as Exceptional Points (EP) [20]. For Hermitian problem involving real parameter, the veering is known to be a consequence of the existence of EP close to the real axis [31].

This paper investigates three eigenvalues representations through Taylor series, Puiseux series and using a combination of analytic auxiliary functions. It is known that using classical perturbation methods to characterize random eigenfrequencies, ie the eigenvalue Taylor series, behave poorly in presence of veering and the effect of EP on the Taylor series radius of convergence is clearly identified. To locate the EP and to compute the higher order coefficient of Puiseux series, the method proposed by Nennig and Perrey-Debain [36] is used. The Puiseux series appear as a good approximation in the vicinity of the EP and successfully describe the modal branches and their topological structure.

As the Puiseux series is centered on the EP, this representation handles a larger variation of the parameter than Taylor series when the computation point is inside a veering area. The relative gain between the two approximations approaches is quantified. Nevertheless, for the application considered in this paper, the presence of an EP for the complex conjugated value of the parameter limit the radius of convergence of the Puiseux. Indeed, the more the veering is pronounced, the more the pair of EPs will be close to the real axis $\left(\mathfrak{I m}\left(\nu^{*}, \bar{\nu}^{*}\right) \rightarrow 0\right)$, and the radius of convergence of the Puiseux series will be limited.

Finally, this work reveals that the analytic auxiliary functions approach provides the largest radius of convergence since these functions are bounded by the existence of EP associated to other eigenvalues of the problem. This representation is robust and allows to significantly reduce the computational cost in comparison with a Monte-Carlo simulation considering the entire structure model.

This work is restricted to a single parameter. More sophisticated approaches are needed to handle multiparamtric perturbations [42, 50] and implicit parameters. Future works will focus on these extansions.

The proposed method could also be particularly relevant for many other applications based on parametric eigenvalue problems like rotating machinery, waveguide dispersion curves computation or to locate instability as squeal or flutter. 


\section{Appendix A. Successive derivative for Hermitian matrix}

For Hermitian problem, if the parameter $\nu$ remains real, the system (6) and the RHS (7) can be modified to retain the symmetry of the initial matrices. Remarking that $\partial_{\lambda} \mathbf{L}=-\mathbf{M}$, the normalization condition given in Eq. (5) can be replaced by the more usual M-norm for generalized eigenvalue problem

$$
\boldsymbol{\Phi}_{i}^{t} \mathbf{M} \boldsymbol{\Phi}_{i}=1
$$

and derived thanks to the Liebnitz' rule

$$
\begin{aligned}
\left(\boldsymbol{\Phi}_{i}^{t} \mathbf{M} \boldsymbol{\Phi}_{i}\right)^{(n)} & =\sum_{k=0}^{n}\left(\begin{array}{l}
n \\
k
\end{array}\right) \boldsymbol{\Phi}_{i}^{(k) t} \mathbf{M} \boldsymbol{\Phi}_{i}^{(n-k)}=0 \\
& =\boldsymbol{\Phi}_{i}^{t} \mathbf{M} \boldsymbol{\Phi}_{i}^{(n)}+\boldsymbol{\Phi}_{i}^{(n) t} \mathbf{M} \boldsymbol{\Phi}_{i}+\sum_{k=1}^{n-1}\left(\begin{array}{c}
n \\
k
\end{array}\right) \boldsymbol{\Phi}_{i}^{(k) t} \mathbf{M} \boldsymbol{\Phi}_{i}^{(n-k)} \\
& =2 \boldsymbol{\Phi}_{i}^{t} \mathbf{M} \boldsymbol{\Phi}_{i}^{(n)}+\sum_{k=1}^{n-1}\left(\begin{array}{l}
n \\
k
\end{array}\right) \boldsymbol{\Phi}_{i}^{(k) t} \mathbf{M} \boldsymbol{\Phi}_{i}^{(n-k)}=2 \boldsymbol{\Phi}_{i}^{t} \mathbf{M} \boldsymbol{\Phi}_{i}^{(n)}+\sum_{k=1}^{\left\lfloor\frac{n-1}{2}\right\rfloor}\left(\begin{array}{c}
n \\
k
\end{array}\right)\left(2-\delta_{\frac{n-1}{2}, k}\right) \boldsymbol{\Phi}_{i}^{(k) t} \mathbf{M} \boldsymbol{\Phi}_{i}^{(n-k)}
\end{aligned}
$$

Here, $\delta_{\frac{n-1}{2}, k}$ is the Kronecker symbol and is equal to one only when $(n-1) / 2$ is equal to $k$ and \lfloor\rfloor is the floor function. The last simplification take into account the symmetry to simplify the summation. This yield the symmetric linear system

$$
\left[\begin{array}{cc}
\mathbf{K}-\lambda_{i} \mathbf{M} & -\mathbf{M} \boldsymbol{\Phi}_{i} \\
-\boldsymbol{\Phi}_{i}^{t} \mathbf{M} & 0
\end{array}\right]\left(\begin{array}{c}
\boldsymbol{\Phi}_{i}^{(n)} \\
\lambda_{i}^{(n)}
\end{array}\right)=\left(\begin{array}{c}
\mathbf{F}_{n}^{\prime} \\
\mathbf{N}_{n}^{\prime}
\end{array}\right)
$$

where

$$
\begin{aligned}
& \mathbf{F}_{n}^{\prime}=-\sum_{k=1}^{n}\left(\begin{array}{l}
n \\
k
\end{array}\right) \mathbf{K}^{(k)} \boldsymbol{\Phi}_{i}^{(n-k)}+\mathbf{M} \sum_{k=1}^{n-1}\left(\begin{array}{l}
n \\
k
\end{array}\right) \lambda_{i}^{(k)} \boldsymbol{\Phi}_{i}^{(n-k)}, \\
& \mathbf{N}_{n}^{\prime}=\frac{1}{2} \sum_{k=1}^{\left\lfloor\frac{n-1}{2}\right\rfloor}\left(\begin{array}{l}
n \\
k
\end{array}\right)\left(2-\delta_{\frac{n-1}{2}, k}\right) \boldsymbol{\Phi}_{i}^{(k) t} \mathbf{M} \boldsymbol{\Phi}_{i}^{(n-k)} .
\end{aligned}
$$

As mention in sec. 2, we consider that the Mass matrix is deterministic to simplify the expression of the RHS. This approach can be view as a generalization of [51] to high order derivative and can take advantage of Cholesky direct solver and symmetric storage in comparison to LU factorization. The price to pay is i) a more costly recurrence relation and ii) the constraint of keeping $\nu$ real. This alternative becomes interesting for big size problem, where CPU time is mainly driven by the matrix factorization.

\section{Appendix B. Puiseux series coefficient computation details}

Once an approximation of $\nu^{*}$ has been found, the computation of the Puiseux series coefficients can be done in two steps. First, the even terms of the Puiseux expansion at $\nu^{*}$ are obtained by matching the Taylor series $T_{g}$, obtained around the point $\nu_{0}$,

$$
T_{g}(\nu)=\sum_{n=0}^{N} b_{n}\left(\nu-\nu_{0}\right)^{n}=\sum_{n=0}^{N} b_{n} \sum_{k=0}^{n}\left(\begin{array}{l}
n \\
k
\end{array}\right)\left(-\nu_{0}\right)^{n-k} \nu^{k},
$$

and the series $T_{g}^{\prime}$ obtained by the substitution of the truncated Puiseux series (11) in Eq. (12), until the order $N$

$$
T_{g}^{\prime}(\nu)=\sum_{n=0}^{N} 2 a_{2 n}\left(\nu-\nu^{*}\right)^{n}=\sum_{n=0}^{N} 2 a_{2 n} \sum_{k=0}^{n}\left(\begin{array}{l}
n \\
k
\end{array}\right)\left(-\nu^{*}\right)^{n-k} \nu^{k} .
$$


Equating each power of $\nu$ allows to produce a set of equations which can be written as a linear system

$$
2 \mathbf{P}\left(\nu^{*}\right) \mathbf{a}_{e}=\mathbf{P}\left(\nu_{0}\right) \mathbf{b} .
$$

It relates the unknown even coefficients of the Puiseux series gathered in the vector $\mathbf{a}_{e}$ to those of the Taylor series collected in $\mathbf{b}$. Each term of the upper triangular matrix $\mathbf{P}(\xi)$ is given by

$$
(\mathbf{P}(\xi))_{k n}= \begin{cases}\left(\begin{array}{l}
n \\
k
\end{array}\right)(-\xi)^{n-k}, & \text { if } n \geq k \\
0, & \text { otherwise. }\end{cases}
$$

Note the matrix is of size $2\left\lfloor\frac{N}{2}\right\rfloor$ which corresponds to the highest order involved.

Finally, the odd terms of the Puiseux series are obtained in a similar manner using the truncated Taylor series $T_{h}$. It is convenient to operate the change of variable $\nu^{\prime}=\nu-\nu^{*}$ so we can write

$$
T_{h}(\nu)=\sum_{n=0}^{N} c_{n}\left(\nu^{\prime}-\left(\nu_{0}-\nu^{*}\right)\right)^{n}=\sum_{n=0}^{N} c_{n} \sum_{k=0}^{n}\left(\begin{array}{l}
n \\
k
\end{array}\right)\left(\nu^{*}-\nu_{0}\right)^{n-k} \nu^{\prime k}=\sum_{k=0}^{N}\left(\mathbf{P}\left(\nu_{0}-\nu^{*}\right) \mathbf{c}\right)_{k} \nu^{\prime k} .
$$

After substitution of the Puiseux series (11) in Eq. 13) and using the multinomial theroem, we get

$$
h(\nu)=4\left(a_{1} \nu^{\prime \frac{1}{2}}+a_{3} \nu^{\prime \frac{3}{2}}+\ldots\right)^{2}=4 \sum_{k=0,1,2, \ldots} \nu^{\prime k} \sum_{n=1,3, \ldots, 2 k-1} a_{n} a_{2 k-n} .
$$

Equating each power of $\nu^{\prime}$ gives explicitly the first coefficient

$$
a_{1}= \pm \frac{1}{2} \sqrt{\left(\mathbf{P}\left(\nu_{0}-\nu^{*}\right) \mathbf{c}\right)_{1}},
$$

where the sign refers to one of two branches of the Puiseux series given in Eq. (11). The others coefficients are obtained iteratively as

$$
a_{2 k-1}=\frac{1}{8 a_{1}}\left\{\left(\mathbf{P}\left(\nu_{0}-\nu^{*}\right) \mathbf{c}\right)_{k}-4 \sum_{n=3,5, \ldots, 2 k-3} a_{n} a_{2 k-n}\right\}, \quad k=2,3, \ldots
$$

\section{References}

[1] L. Zadeh, Fuzzy sets as a basis for a theory of possibility, Fuzzy Sets Syst. 100 (1) (1999) $9-34$. doi:10.1016/S0165-0114(99)80004-9

[2] P. Ladevèze, G. Puel, T. Romeuf, Lack of knowledge in structural model validation, Comput. Methods in Appl. Mech. Eng. 195 (37) (2006) 4697 - 4710. doi:10.1016/j.cma.2005.10.017.

[3] S. Daouk, F. Louf, O. Dorival, L. Champaney, On the lack-of-knowledge theory for low and high values of uncertainties, in: 2nd International Symposium on Uncertainty Quantification and Stochastic Modeling, Uncertainties, Rouen, France, 2014, p. 1.

URL https://hal . archives-ouvertes.fr/hal-01023550

[4] S. Daouk, F. Louf, O. Dorival, L. Champaney, S. Audebert, Uncertainties in structural dynamics: overview and comparative analysis of methods, Mech.\& Ind. 16 (4) (2015) 404. doi:10.1051/meca/ 2015010.

[5] C. Soize, Stochastic modeling of uncertainties in computational structural dynamics - recent theoretical advances, J. Sound Vib. 332 (10) (2013) 2379-2395. doi:10.1016/j·jsv.2011.10.010.

[6] G. Stefanou, The stochastic finite element method: Past, present and future, Comput. Methods in Appl. Mech. Eng. 198 (9) (2009) 1031 - 1051. doi:10.1016/j.cma.2008.11.007. 
[7] M. Shinozuka, C. J. Astill, Random eigenvalue problems in structural analysis, AIAA J. 10 (4) (1972) 456-462. doi:10.2514/3.50119.

[8] M. Papadrakakis, A. Kotsopulos, Parallel solution methods for stochastic finite element analysis using monte carlo simulation, Comput. Methods in Appl. Mech. Eng. 168 (1) (1999) 305-320. doi:10.1016/ S0045-7825(98) 00147-9.

[9] S. Adhikari, M. Friswell, Random matrix eigenvalue problems in structural dynamics, Int. J. Numer. Meth. Engng. 69 (3) (2007) 562-591. doi:10.1002/nme.1781.

[10] J. D. Collins, W. T. Thomson, The eigenvalue problem for structural systems with statistical properties, AIAA J. 7 (4) (1969) 642-648. doi:10.2514/3.5180.

[11] B. V. den Nieuwenhof, J.-P. Coyette, Modal approaches for the stochastic finite element analysis of structures with material and geometric uncertainties, Comput. Methods in Appl. Mech. Eng. 192 (33) (2003) 3705 - 3729. doi:10.1016/S0045-7825(03)00371-2.

[12] R. Ghanem, P. D. Spanos, Polynomial chaos in stochastic finite elements, J. Appl. Mech. 57 (1) (1990) 197-202. doi:10.1115/1.2888303.

[13] R. Ghanem, D. Ghosh, Efficient characterization of the random eigenvalue problem in a polynomial chaos decomposition, Int. J. Numer. Methods Eng. 72 (4) (2007) 486-504. doi:10.1002/nme.2025.

[14] G. Blatman, B. Sudret, Adaptive sparse polynomial chaos expansion based on least angle regression, J. Comput. Phys. 230 (6) (2011) 2345 - 2367. doi:10.1016/j.jcp.2010.12.021.

[15] J.-J. Sinou, J. Didier, B. Faverjon, Stochastic non-linear response of a flexible rotor with local nonlinearities, Int. J. Non Linear Mech. 74 (2015) 92 - 99. doi:10.1016/j.ijnonlinmec.2015.03.012.

[16] N. Wiener, The homogeneous chaos, Am. J. Math. 60 (4) (1938) 897-936.

[17] G. Blatman, B. Sudret, An adaptive algorithm to build up sparse polynomial chaos expansions for stochastic finite element analysis, Probab. Eng. Mech. 25 (2) (2010) 183 - 197. doi:10.1016/j. probengmech.2009.10.003

[18] A. Gallina, L. Pichler, T. Uhl, Enhanced meta-modelling technique for analysis of mode crossing, mode veering and mode coalescence in structural dynamics, Mech. Syst. Sig. Process. 25 (7) (2011) 2297 2312. doi:10.1016/j.ymssp.2011.02.020.

[19] V. Dubourg, B. Sudret, F. Deheeger, Metamodel-based importance sampling for structural reliability analysis, Probab. Eng. Mech. 33 (2013) 47 - 57. doi:10.1016/j.probengmech.2013.02.002.

[20] T. Kato, Perturbation Theory for Linear Operators, 2nd edition, Springer-Verlag, Berlin, Heidelberg, 1980, p. 623pp. doi:10.1007/978-3-642-66282-9.

[21] P. Nair, A. Keane, An approximate solution scheme for the algebraic random eigenvalue problem, J. Sound Vib. 260 (1) (2003) 45 - 65. doi:10.1016/S0022-460X(02) 00899-4.

[22] M. Kamiński, The Stochastic Perturbation Method for Computational Mechanics: Practical Applications in Science and Engineering, John Wiley \& Sons, Hoboken, New Jersey, USA, 2013.

[23] W. K. Liu, T. Belytschko, A. Mani, Random field finite elements, Int. J. Numer. Methods Eng. 23 (10) (1986) 1831-1845. doi:10.1002/nme.1620231004.

[24] M. Kamiński, Generalized stochastic perturbation technique in engineering computations, Math. Comput. Modell. 51 (3-4) (2010) 272-285. doi:j.mcm.2009.08.014.

[25] M. Ghienne, C. Blanzé, L. Laurent, Stochastic model reduction for robust dynamical characterization of structures with random parameters, C.R. Mec. 345 (12) (2017) 844 - 867. doi:10.1016/j.crme. 2017.09 .006 . 
[26] H. Baumgärtel, Analytic perturbation theory for matrices and operators, Vol. 15, Birkhauser Verlag, Basel,, 1985.

[27] L. Xiong, B. Nennig, Y. Aurégan, W. Bi, Sound attenuation optimization using metaporous materials tuned on exceptional points, J. Acoust. Soc. Am. 142 (4) (2017) 2288 - 2297. doi:10.1121/1.5007851.

[28] R. O. Akinola, M. A. Freitag, A. Spence, The computation of Jordan blocks in parameter-dependent matrices, IMA J. Numer. Anal. 34 (3) (2014) 955-976. doi:10.1093/imanum/drt028.

[29] A. Seyranian, A. Mailybaev, Multiparameter stability theory with mechanical applications, Vol. 13, World Scientific, 2003.

[30] C. Pierre, Mode localization and eigenvalue loci veering phenomena in disordered structures, J. Sound Vib. 126 (3) (1988) 485-502. doi:10.1016/0022-460X(88)90226-X.

[31] L. Dieci, A. Papini, A. Pugliese, A. Spadoni, Continuous Decompositions and Coalescing Eigenvalues for Matrices Depending on Parameters, Vol. 2082 of In: Current Challenges in Stability Issues for Numerical Differential Equations. Lecture Notes in Mathematics, Springer, 2014, pp. 173-264. doi: 10.1007/978-3-319-01300-8_4

[32] E. Manconi, B. Mace, Veering and strong coupling effects in structural dynamics, J. Vib. Acoust. 139 (2) (2017) 021009. doi:10.1115/1.4035109

[33] X. Liu, Behavior of derivatives of eigenvalues and eigenvectors in curve veering and mode localization and their relation to close eigenvalues, J. Sound Vib. 256 (3) (2002) 551 - 564. doi:10.1006/jsvi. 2002.5010 .

[34] J. L. Du Bois, S. Adhikari, N. Lieven, On the quantification of eigenvalue curve veering: a veering index, J. Appl. Mech. 78 (4) (2011) pp. 041007-1 - 041007-8. doi:10.1115/1.4003189.

[35] C. M. Bender, PT Symmetry: In Quantum and Classical Physics, World Scientific Publishing, 2018.

[36] B. Nennig, E. Perrey-Debain, A high order continuation method to locate exceptional points and to compute puiseux series with applications to acoustic waveguides, J. Comp. Phys. (Accepted for publication.).

[37] M. Friswell, The derivatives of repeated eigenvalues and their associated eigenvectors, J. Vib. Acoust. 118 (3) (1996) 390-397. doi:10.1115/1.2888195.

[38] M. Triantafyllou, G. Triantafyllou, Frequency coalescence and mode localization phenomena: A geometric theory, J. Sound Vib. 150 (3) (1991) 485 - 500. doi:10.1016/0022-460X(91)90899-U.

[39] A. L. Andrew, K.-W. E. Chu, P. Lancaster, Derivatives of eigenvalues and eigenvectors of matrix functions, SIAM J. Matrix Anal. Appl. 14 (4) (1993) 903-926. doi:10.1137/0614061.

[40] D. V. Murthy, R. T. Haftka, Derivatives of eigenvalues and eigenvectors of a general complex matrix, Int. J. Num. Meth. Eng. 26 (2) (1988) 293-311. doi:10.1002/nme.1620260202.

[41] E. T. Whittaker, G. N. Watson, A course of modern analysis, Cambridge university press, 1965.

[42] A. A. Mailybaev, Computation of multiple eigenvalues and generalized eigenvectors for matrices dependent on parameters, Numer. Linear Algebra Appl. 13 (5) (2006) 419-436. doi:10.1002/nla.471.

[43] S. Christiansen, P. A. Madsen, On truncated taylor series and the position of their spurious zeros, Appl. num. math. 56 (1) (2006) 91-104. doi:10.1016/j.apnum.2005.02.009

[44] A. Luongo, Eigensolutions of perturbed nearly defective matrices, J. Sound Vib. 185 (3) (1995) 377-395. doi:10.1006/jsvi.1995.0387. 
[45] A. P. Seyranian, O. N. Kirillov, A. A. Mailybaev, Coupling of eigenvalues of complex matrices at diabolic and exceptional points, J. Phys. A 38 (8) (2005) 1723. doi:10.1088/0305-4470/38/8/009.

[46] E. Hernández, A. Jáuregui, A. Mondragón, Energy eigenvalue surfaces close to a degeneracy of unbound states: Crossings and anticrossings of energies and widths, Phys. Rev. E 72 (2) (2005) 026221. doi: 10.1103/PhysRevE.72.026221.

[47] H. Cartarius, J. Main, G. Wunner, Exceptional points in the spectra of atoms in external fields, Phys. Rev. A 79 (2009) 053408. doi:10.1103/PhysRevA.79.053408

[48] R. Uzdin, R. Lefebvre, Finding and pinpointing exceptional points of an open quantum system, J. Phys. B 43 (23) (2010) 235004. doi:10.1088/0953-4075/43/23/235004.

[49] A. Welters, On explicit recursive formulas in the spectral perturbation analysis of a Jordan block, SIAM J. Matrix Anal. Appl. 32 (1) (2011) 1-22. doi:10.1137/090761215

[50] A. P. Seyranian, A. A. Mailybaev, Interaction of eigenvalues in multi-parameter problems, J. Sound Vib. 267 (5) (2003) 1047-1064. doi:10.1016/S0022-460X(03)00360-2.

[51] I.-W. Lee, G.-H. Jung, An efficient algebraic method for the computation of natural frequency and mode shape sensitivities-Part i. distinct natural frequencies, Comput. Struct. 62 (3) (1997) 429 - 435. doi:10.1016/S0045-7949(96)00206-4. 